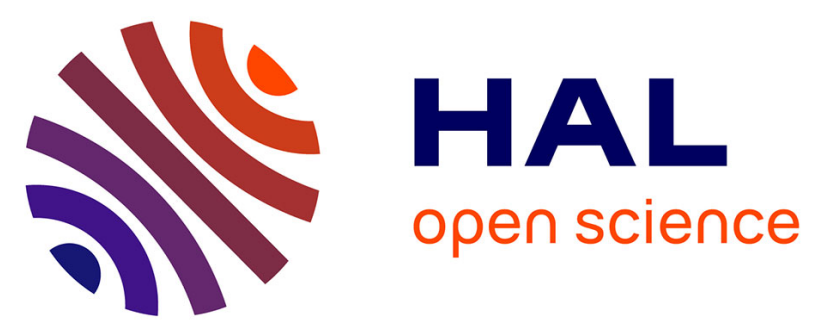

\title{
Pks5-recombination-mediated surface remodelling in Mycobacterium tuberculosis emergence
}

Eva C. Boritsch, Wafa Frigui, Alessandro Cascioferro, Wladimir Malaga, Gilles Etienne, Françoise Laval, Alexandre Pawlik, Fabien Le Chevalier, Mickael Orgeur, Laurence Ma, et al.

\section{To cite this version:}

Eva C. Boritsch, Wafa Frigui, Alessandro Cascioferro, Wladimir Malaga, Gilles Etienne, et al.. Pks5recombination-mediated surface remodelling in Mycobacterium tuberculosis emergence. Nature Microbiology, 2016, 1 (février 2016), 10.1038/nmicrobiol.2015.19 . pasteur-01265519

\section{HAL Id: pasteur-01265519}

https://hal-pasteur.archives-ouvertes.fr/pasteur-01265519

Submitted on 1 Feb 2016

HAL is a multi-disciplinary open access archive for the deposit and dissemination of scientific research documents, whether they are published or not. The documents may come from teaching and research institutions in France or abroad, or from public or private research centers.
L'archive ouverte pluridisciplinaire HAL, est destinée au dépôt et à la diffusion de documents scientifiques de niveau recherche, publiés ou non, émanant des établissements d'enseignement et de recherche français ou étrangers, des laboratoires publics ou privés.

\section{(1) (1) $\$$}

Distributed under a Creative Commons Attribution - NonCommercial - NoDerivatives 44.0 
$1 \quad$ pks5-recombination-mediated surface remodelling in Mycobacterium tuberculosis

2 emergence

3

4 Eva C. Boritsch ${ }^{1}$, Wafa Frigui ${ }^{1}$, Alessandro Cascioferro ${ }^{1}$, Wladimir Malaga ${ }^{2,3}$, Gilles

5 Etienne $^{2,3}$, Françoise Laval ${ }^{2,3}$, Alexandre Pawlik ${ }^{1}$, Fabien Le Chevalier ${ }^{1,5}$, Mickael Orgeur ${ }^{1}$,

6 Laurence $\mathrm{Ma}^{4}$, Christiane Bouchier ${ }^{4}$, Timothy P. Stinear ${ }^{6}$, Philip Supply ${ }^{7}$, Laleh Majlessi ${ }^{1}$,

7 Mamadou Daffé, ${ }^{2,3}$, Christophe Guilhot ${ }^{2,3,{ }^{*}}$ and Roland Brosch ${ }^{1, *}$.

8

$9 \quad{ }^{1}$ Institut Pasteur, Unit for Integrated Mycobacterial Pathogenomics, 75015 Paris, France;

$10{ }^{2}$ CNRS, IPBS (Institut de Pharmacologie et de Biologie Structurale), 31000 Toulouse, France;

$11{ }^{3}$ Université de Toulouse, UPS, IPBS, 31000 Toulouse, France;

$12{ }^{4}$ Institut Pasteur, PF1-Plate-Forme Génomique, Paris, France;

$13{ }^{5}$ University Paris Diderot, Sorbonne Paris Cité, Cellule Pasteur, Paris, France

$14{ }^{6}$ Department of Microbiology and Immunology, University of Melbourne, Parkville,

15 Victoria, Australia.

$16{ }^{7}$ Inserm U1019, CNRS UMR8204, Université de Lille, Institut Pasteur de Lille, Center for 17 Infection and Immunity, 59000 Lille, France.

18

19

*Corresponding authors: Christophe.Guilhot@ipbs.fr and roland.brosch@pasteur.fr

20 
21 Mycobacterium tuberculosis is a major, globally spread, aerosol-transmitted human pathogen, 22 thought to have evolved by clonal expansion from a Mycobacterium canettii-like progenitor. 23 In contrast, extant M. canettii strains are rare, genetically diverse and geographically 24 restricted mycobacteria of only marginal epidemiological importance. Here we show that the 25 contrasting evolutionary success of these two groups is linked to loss of lipooligosaccharide 26 (LOS) biosynthesis and subsequent morphotype changes. Spontaneous smooth-to-rough $M$. 27 canettii variants were found mutated in the polyketide-synthase-encoding pks 5 locus and 28 deficient in LOS synthesis; a phenotype restored by complementation. Importantly, these rough variants showed altered host-pathogen interaction and increased virulence in cellularand animal-infection models. In one variant, LOS deficiency occurred via homologous recombination between two pks5 copies and removal of the intervening acyltransferaseencoding gene. The resulting single pks5 configuration is similar to that fixed in $M$.

33 tuberculosis, known to lack LOS. Our results suggest that pks5-recombination-mediated 34 bacterial surface remodelling increased virulence, driving evolution from putative generalist 35 mycobacteria towards professional pathogens of mammalian hosts. 
Tuberculosis is a major human infectious disease. Although many aspects of the diseasecausing potential of its etiological agent Mycobacterium tuberculosis are known ${ }^{1}$, our understanding is scant of the molecular events that favoured its evolutionary success as one of the most widely distributed human pathogens. New insights into this question are important for uncovering mechanisms of pathogenesis and new drug targets ${ }^{2}$. Strains of the closely related and phylogenetically early branching Mycobacterium canettii, also named smooth tubercle bacilli (STB) ${ }^{3}$ are powerful resources to investigate the evolution of M. tuberculosis and the M. tuberculosis complex (MTBC) ${ }^{3}$. The first strain of M. canettii was isolated by Georges Canetti in 1969 and since then less than 100 isolates have been described, most of which have been isolated from tuberculosis patients with a connection to the Horn of Africa ${ }^{4-}$ 7. Despite their geographic restriction, M. canettii strains show much greater genetic variability and are less virulent/persistent than M. tuberculosis. Genome comparisons suggest that $M$. tuberculosis evolved by clonal expansion from a pool of $M$. canettii-like tubercle bacilli through the gain of virulence and persistence mechanisms ${ }^{3,8,9}$. While some genomic differences were found to be specific for a single $M$. canettii strain, apparently due to isolated horizontal gene transfer (e.g. the $m c e 5$ operon $^{3}$ or the eptABCD operon ${ }^{10}$, present exclusively in strain STB-J), others are conserved throughout all $M$. canettii strains as a result of phylogenetic ancestry (e.g. cobF, present in M. canettii and deleted from the MTBC) ${ }^{3}$.

Here we investigated phenotypic differences between M. canettii and M. tuberculosis and focused on the unique, conserved, smooth (S) colony morphotype of M. canettii, as opposed to the rough (R) morphotype of MTBC members. Previously, S-morphotypes of nontuberculous mycobacterial species such as Mycobacterium avium ${ }^{11}$, Mycobacterium abscessus $^{12}$, Mycobacterium kansasii ${ }^{13}$ or Mycobacterium marinum ${ }^{14}$ have been found to be less virulent than R-morphotypes, raising the question of whether the highly conserved $M$. tuberculosis R-morphotype might have been the result of evolutionary selection, based on host-pathogen interactions favouring a more virulent or persistent phenotype. In other mycobacterial species, $S / R$ variation is often attributed to different kinds of cell surface glycolipids, such as glycopeptidolipids (GPL) for M. avium $^{15}$ and M. abscessus ${ }^{16,17}$ or lipooligosaccharides (LOS) for M. kansasii ${ }^{13}$ and M. marinum $^{14,18,19}$. Insights from earlier studies for $M$. canettii have remained abstruse as no specific lipid exclusively present in the Smorphotype has been identified ${ }^{20}$, nor has the genetic basis for morphotype variation been determined ${ }^{4}$.

Building on recent data from several $M$. canettii genomes ${ }^{3}$, here we studied smooth and spontaneously converted R-variants of two different M. canettii strains, STB-K (CIPT 
140070010) and STB-I (CIPT 140070007) ${ }^{3}$, hereafter named $\mathrm{K}_{\mathrm{S}} \mathrm{R}_{\mathrm{R}}$ and $\mathrm{I}_{\mathrm{S} / \mathrm{R}}$, respectively. We used whole genome sequencing (WGS) and identified differences in genes of the pks5 locus, which in Mycobacterium smegmatis, M. marinum or M. kansasii are implicated in LOS biosynthesis $^{14,21-23}$. In this report we uncover the mechanisms underlying the S-to-R morphology change of tubercle bacilli and explore the biological consequences with emphasis on the patho-evolution of $M$. tuberculosis.

\section{RESULTS}

\section{Genome comparison of M. canettii S- and R-variants}

During passaging of different smooth $M$. canettii strains, we observed spontaneous Rmorphotype variants for two M. canettii strains (I and K). These R-variants were re-passaged three times on solid medium to ensure stable phenotypes and subjected to Illumina-based WGS. The results obtained pointed to morphotype-linked alterations in the pks 5 locus of $M$. canettii S/R variants (Supplementary Tables 1 and 2, Supplementary Note), which was of particular interest given suggested roles for Pks5 polyketide synthases in morphotype changes and LOS production in different mycobacterial species ${ }^{14,21}$. In the LOS-producers $M$. marinum $^{24}$ and $M$. kansasii ${ }^{25}$, the Pks5-encoding locus harbours two proximal pks5 homologs separated by one or more interspaced pks5-associated gene(s) (Fig. 1A). The genome of $M$. canettii STB- $\mathrm{A}^{3}$ also shows a twin-pks 5 configuration similar to M. marinum. In contrast $M$. tuberculosis, which does not produce LOS only has a single pks5 and lacks a full-length pap ortholog (Fig. 1A).

\section{Recombination of two pks 5 genes in $M$. canettii $\mathrm{K}_{\mathrm{R}}$ and $M$. tuberculosis}

To characterize the pks5 locus in strains $\mathrm{I}_{\mathrm{S} / \mathrm{R}}, \mathrm{K}_{\mathrm{S} / \mathrm{R}}$ and M. tuberculosis $\mathrm{H} 37 \mathrm{Rv}$ we performed long-range PCR using pks5-flanking primers, yielding amplicons of ca. $15 \mathrm{~kb}$ for both the Sand R-variants of strain I, and $6 \mathrm{~kb}$ for M. tuberculosis H37Rv, corresponding to the twin- and single-pks5 configuration, respectively (Fig. 1 A, B). For strain K, however, the prevalent amplicon size of the S-variant was $15 \mathrm{~kb}$, while the size for the R-variant was $6 \mathrm{~kb}$, which was surprising because the previously released STB-K genome sequence assembly ${ }^{3}$ indicated only a single pks5. The PCR result suggests that the previous WGS-based genome assembly of strain K (NC_019951) contained a misassembly in that region probably due to high sequence identity ( $>94 \%$ ) between the two pks5 genes. Similarly, we also confirmed the presence of two pks5 genes by long-range PCR amplicon-sequencing for $M$. canettii strains D and $\mathrm{J}$, for which previously only one pks5 was indicated in the released genome sequences NC_019950 
104 and NC_019952, respectively (Supplementary Fig. 1). Hence, the results obtained for S- and

$105 \mathrm{R}$-variants of strain $\mathrm{K}$ suggest that $\mathrm{K}_{\mathrm{S}}$ contains two pks5 genes, flanking a gene (pap) coding 106 for a putative polyketide-synthase-associated acyltransferase ${ }^{26}$, whereas $\mathrm{K}_{\mathrm{R}}$ only harbours a 107 single $p k s 5$ and no interspaced pap gene.

108 These findings were supported by results obtained by alignment to the reference 109 sequences of M. canettii STB-A and M. tuberculosis H37Rv (Supplementary Fig. 2 and 110 Supplementary Note). This analysis suggested that homologous recombination in strain $K_{R}$ 111 occurred at a site that left intact the typical polyketide-synthase domain structures of Pks $5^{26,27}$ 112 (Fig. 1). The observed pks5 configuration in $\mathrm{K}_{\mathrm{R}}$ can thus serve as a model for the situation 113 found in $M$. tuberculosis, where two former pks 5 genes also seem to have coalesced into a 114 single pks5 gene. In the latter case, the proposed recombination event also left the domain 115 organisation of Pks5 intact, although the site of recombination was apparently different from 116 the site observed for $K_{R}$ (Fig. 1 and Supplementary Fig. 2B). Further comparisons among 117 different MTBC members $^{9}$ showed $>99.93 \%$ sequence identity of the single pks 5 gene in 118 these strains, suggesting a unique recombination event in the most recent common ancestor of 119 the MTBC after the separation from an M. canettii-like progenitor.

120 Finally, for strain $\mathrm{I}$, amplicon-sequencing of the pks 5 locus of $\mathrm{I}_{\mathrm{R}}$ and $\mathrm{I}_{\mathrm{S}}$ confirmed the 121 presence of pap and two pks5 copies in both morphotypes. However, alignment of amplicon122 derived sequences indicated the presence of multiple non-synonymous SNPs in both pks 5 123 genes (6 in pks5-2 and 3 in pks5-1) for the R-variant (Supplementary Fig. 3A), which likely 124 resulted from recombination of the ketosynthase domain regions of the pks5 copies 125 (Supplementary Fig. 3B). From these data we predicted that these changes likely caused the 126 R-morphotype of $\mathrm{I}_{\mathrm{R}}$.

\section{Pap and Pks5-2 restore the S-morphotype in $M$. canettii}

129 To test if changes in the pks5 locus were responsible for the $\mathrm{S} / \mathrm{R}$ variations in $M$. canettii, we 130 transformed strains $\mathrm{K}_{\mathrm{R}}$ and $\mathrm{I}_{\mathrm{R}}$, as well as M. tuberculosis $\mathrm{H} 37 \mathrm{Rv}$ with the integrating cosmid 131 C9, selected from an M. canettii STB-A large-fragment genomic library constructed for this 132 purpose in pYUB412. This vector integrates into the attB site located in the $g l y V$-tRNA of 133 mycobacterial genomes ${ }^{28}$. The $30.5 \mathrm{~kb}$ insert of cosmid C9 spans the pks 5 locus of STB-A 134 (Supplementary Fig. 4). Fig. 2 shows that recombinant $M$. canettii strains $\mathrm{I}_{\mathrm{R}}: \mathrm{C} 9$ and $\mathrm{K}_{\mathrm{R}}: \mathrm{C} 9$ 135 regained S-morphology that was indistinguishable from their respective S-variants. To refine 136 the region required for S-morphotype restitution, we isolated a second cosmid, named H6 that 137 contains full-length pap and pks5-2 but a 3'-truncated pks5-1 (Supplementary Fig. 4). 
138 Transformation of $I_{R}$ and $K_{R}$ with $\mathrm{H} 6$ yielded smooth colonies for both $I_{R}:: H 6$ and $K_{R}:: H 6$ 139 (Supplementary Fig. 5A).

140 Complementation with a pks5-2 single gene expression construct restored an S141 morphotype for strain $\mathrm{I}_{\mathrm{R}}:: p k s 5-2$, but not for $\mathrm{K}_{\mathrm{R}}:: p k s 5-2$ (Supplementary Figs. 5B and 5C). 142 Moreover, transformation with pap was unable to restore smooth colonies in either of the 143 strains despite appropriate protein expression (Supplementary Figs. 5B and 5D). Together, the 144 complementation experiments suggest that pks5-2 and pap are necessary for the S145 morphotype in M. canettii.

146 In contrast, R-morphology of $M$. tuberculosis $\mathrm{H} 37 \mathrm{Rv}$ remained unchanged upon 147 transformation with cosmid C9 (Fig. 2A) despite correct genomic integration of the cosmid 148 (Fig. 3). Similarly, transformation of other MTBC lineage members with C9 did not yield S149 morphotypes (Supplementary Fig. 6). These results suggest that apart from an appropriate 150 pks5-pap locus, additional genes intervene in the formation of S-morphotypes in tubercle 151 bacilli.

\section{M. canettii strains synthesize LOS}

154 Analysis of total lipid extracts from a large panel of $M$. canettii strains by thin-layer chromatography (TLC) showed glycoconjugate spots, with TLC mobilities similar to that of LOS from M. canettii reference strain CIPT-140010059 (STB-A or M. canettii strain A) ${ }^{29}$ in all tested strains. Substantial differences in the quantity produced by each strain were observed (Fig. 4A). In contrast, no such glycoconjugates were detected in M. tuberculosis H37Rv, consistent with the long-standing knowledge that M. tuberculosis does not synthesize LOS (Fig. 4A) ${ }^{30}$. Purification and MALDI-TOF mass spectrometry analysis of LOS from $M$. canettii strains $\mathrm{A}, \mathrm{K}_{\mathrm{S}}$ and $\mathrm{I}_{\mathrm{S}}$ revealed pseudomolecular-ion mass peaks at $\mathrm{m} / \mathrm{z} 2530,2572$, and 2614 for LOS from strain $\mathrm{K}_{\mathrm{S}}$ (Fig. 4B) and strain $\mathrm{A}^{29}$, whereas the pattern of strain $\mathrm{I}_{\mathrm{S}}$ showed peaks at $\mathrm{m} / \mathrm{z} 2516,2558$ and 2600 (Fig. 4B), consistent with the different mobility on TLC (Figs. 4A and 4C). The 14 mass-unit difference indicates strain-specific structural differences, likely due to the absence of one methyl-group from the carbohydrate or the lipid moiety. The structure of the simplest carbohydrate domain of the M. canettii LOS is 2-O-Me$\alpha$-L-Fucp (1->3)- $\beta$-D-Glcp(1->3)-2-O-Me- $\alpha$-L-Rhap (1->3)-2-O-Me- $\alpha-L-R h a p(1->3)-\beta-D-$

168 Glcp(1->3)-4-O-Me- $\alpha$-L-Rhap(1->3)-6-O-Me- $\alpha-D-G l c(1<->1)-t r i-O-a c y l-\alpha-D-G l c$ (Fig. 4D). 169 This octaglycosyl unit is usually further glycosylated by an incompletely defined $N$-acyl 170 derivative of a 4-amino-4,6-dideoxy-Gal $p$ residue to generate a second nonasaccharide171 containing glycolipid (LOS 9) (Fig. 4D) ${ }^{29}$. The MALDI-TOF mass spectra of LOS purified 
172 from strains $\mathrm{K}$ and $\mathrm{A}$ are consistent with the LOS 9 structure. Of note, similar patterns of ion 173 mass peaks were detected in spectra of LOS purified from M. canettii strains D, E, F, G, L, 174 and $\mathrm{H}$, while LOS of strain $\mathrm{J}$ showed a 14 mass difference, similar to strain $\mathrm{I}_{\mathrm{S}}$ (Supplementary 175 Fig. 7).

176 Rough $M$. canettii variants I and $\mathrm{K}$ are deficient in LOS biosynthesis

177 Having established that all tested M. canettii strains synthesized LOS, we next compared the 178 production of these glycoconjugates in the R-variants of $\mathrm{I}$ and $\mathrm{K}$. As predicted, $\mathrm{I}_{\mathrm{R}}$ was 179 severely impaired in LOS production with only trace amounts detected, and LOS was 180 undetectable in $\mathrm{K}_{\mathrm{R}}$ (Fig. 4C and Supplementary Fig. 8). No other differences in polyketide181 derived-lipids were observed between the S- and R-variants (Supplementary Fig. 9). 182 Complementation with cosmid C9 restored LOS biosynthesis in recombinant I and K strains 183 (Fig. 4C), whereas recombinant expression of pks5-2 alone restored the LOS profile in $184 \mathrm{I}_{\mathrm{R}}:$ :pks5-2, but not in $\mathrm{K}_{\mathrm{R}}:$ :pks5-2 (Supplementary Fig. 10A), likely due to the absence of full185 length pap in $\mathrm{K}_{\mathrm{R}}:: p k s 5-2$. Indeed, based on current knowledge on acyl-trehalose biosynthesis in $M$. tuberculosis $^{31,32}$, a pap-encoded acyltransferase is needed to catalyze the transfer of 187 Pks-produced polymethyl-branched fatty acids onto trehalose.

188 We also tested LOS production in the MTBC strains complemented with cosmid C9, 189 despite unaffected R-morphotype. LOS was not detected in any of the recombinant $M$. 190 tuberculosis strains (Supplementary Fig. 10B), suggesting that apart from pks 5 recombination 191 and pap deletion, in the MTBC additional loss-of-function mutations or insertions/deletions 192 (indels) have occurred in adjacent genes of the LOS-encoding locus that are not covered by cosmid C9 (Supplementary Tables 3 and 4). These results establish that point mutations or recombination/deletion events in the core pks5-pap locus of $M$. canettii strains $\mathrm{I}_{\mathrm{R}}$ and $\mathrm{K}_{\mathrm{R}}$ led to LOS deficiency and R-morphology. The results also explain the R-morphotype of $M$. tuberculosis and open new perspectives for detailed research on the functions of pks5197 adjacent genes.

\section{Increased fitness and virulence of R-variants in cellular and animal models}

200 Given the identified altered cell surface structure associated with S/R morphology, the question arose whether these changes had an impact on host-pathogen interactions. We thus undertook a series of infection experiments, starting with tests of intracellular replication, a hallmark of mycobacterial virulence. Differentiated human THP-1 macrophages were infected with Sauton-grown $M$. canettii strains $\mathrm{K}_{\mathrm{S}}, \mathrm{K}_{\mathrm{R}}$ and $\mathrm{K}_{\mathrm{R}}:$ :C9, as well as $\mathrm{K}_{\mathrm{R}}:$ :vector-control, up to 
205 five days. While no intracellular growth was observed for $\mathrm{K}_{S}$ and $\mathrm{K}_{\mathrm{R}}: \mathrm{C}$, the R-morphotypes

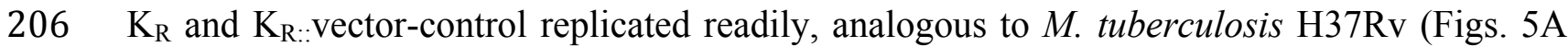
207 and 5B). Similar results were obtained when human peripheral blood monocyte-derived macrophages from healthy donors were infected with the different morphotypes 209 (Supplementary Fig. 11A), confirming that the increased fitness advantage of the R210 morphotypes was a general phenomenon in human macrophages and not just in THP-1 cells. 211 For murine-derived RAW macrophages a similar trend, but without significant difference was 212 observed (Supplementary Fig. 11B).

213 To test whether an increased fitness advantage could also be observed in vivo, we first 214 infected SCID mice intravenously with $1 \times 10^{6} \mathrm{CFU}$ of each morphotype. Mice infected with M. tuberculosis $\mathrm{H} 37 \mathrm{Rv}$ or M. canettii $\mathrm{K}_{\mathrm{R}}$ had a median survival of 16 and 24 days pi, respectively, while mice infected with strains $\mathrm{K}_{\mathrm{S}}$ and $\mathrm{K}_{\mathrm{R}}: \mathrm{C} 9$ had a longer survival time; on average 31 and 36 days, respectively (Fig. 5C). Higher virulence of R-morphotypes was also observed in the sensitive guinea pig model, using low-dose aerosol infection (Fig. 5D). While in this highly susceptible model, the S-variants of $M$. canettii were already substantially virulent and able to replicate in infected animals, the ratios of the bacterial burden in the lungs at day 42 relative to day 1 were significantly higher for strain $K_{R}$ than those for the Smorphotypes. Interestingly, for animals infected with $\mathrm{K}_{\mathrm{R}}:: \mathrm{C} 9$ (Fig. 2) we observed more than half of the colonies showing a re-converted R-morphotype, which was never observed in any of our previous in vitro experiments, suggesting possible reversion of the usually very stable pYUB412-based integrated genetic complementation ${ }^{33}$, due to in vivo fitness advantages of Rmorphotypes during the long-duration in vivo infection assays.

To further investigate the host-pathogen interaction of $\mathrm{S}$ - and R-variants, we characterized the inflammatory responses induced in infected phagocytes, as recently performed for S- and R-forms of M. abscessus $^{34,35}$. As shown in Figs. 5E and 5F, a potential LOS-dependent anti-inflammatory effect was observed in $M$. canettii-infected bone-marrow derived dendritic cells (BM-DCs) of C57BL/6 mice. Strains $\mathrm{K}_{\mathrm{S}}$ and $\mathrm{K}_{\mathrm{R}}: \mathrm{C}$ ( 9 induced significantly lower secretion of NF-кB-dependent inflammatory cytokines IL-6 and IL-12p40 than $K_{R}$. Results with these two cytokines, known to be induced in response to infection with M. tuberculosis $^{36}$, were also representative for TNF- $\alpha$ and NF- $\mathrm{kB}-d e p e n d e n t$ CCL5 and CXCL10 chemokines (Supplementary Fig. 12). In our experimental conditions, most of the responses were dependent on interaction via Toll-like receptor 2 (TLR-2). Smooth variants showed reduced interaction with this crucial innate immune sensor of numerous mycobacterial ligands (Figs. 5 G, 5H and Supplementary Fig. 13). Presence or absence of 
239 LOS thus seems to strongly influence the complex pattern of host-tubercle bacilli interaction, 240 with likely consequences for virulence and pathogenesis.

\section{DISCUSSION}

243 The evolutionary transition for a bacterial population from low-virulence/high genetic 244 diversity towards high-virulence/low genetic diversity is well established for some major 245 human pathogens such as Yersinia pseudotuberculosis vs Yersinia pestis or Salmonella 246 enteritidis vs Salmonella typhi ${ }^{37}$. In contrast, the specific genetic changes that led to the emergence of M. tuberculosis are less well understood. Here we have exploited the ancestralstate characteristics of $M$. canettii to address this gap in our knowledge ${ }^{3,6,9}$. We show that the transition of $M$. tuberculosis from likely generalist to obligate pathogen was associated with a dramatic change in mycobacterial surface glycolipid composition. The S-to-R morphotype variation we have studied here has been linked with higher virulence in opportunistic pathogenic mycobacteria ${ }^{11-14}$ and variation in LOS content has been shown to account for morphology differences in $M$. kansasii $^{13}$ or $M$. marinum ${ }^{14,18,19}$. However, earlier studies on $M$. canettii colony morphology have provided inconclusive results. While a study from van Soolingen and coworkers found differences in LOS content between S/R colony variants of an $M$. canettii strain isolated from a 2-year-old Somali child ${ }^{4}$, Lemassu and colleagues did not observe a correlation between LOS content and colony morphology in various strains tested ${ }^{20}$, likely because the used strains were poor LOS producers with intermediate morphotypes. In this current work we resolve these ambiguities and clearly link LOS biosynthesis with the smooth colony morphotype. We also provide a genetic mechanism for the phenotype that helps explain the evolution of the tubercle bacilli. Our results indicate that recombination of two pks5 genes associated with deletion of the interspersed pap gene evidently was a molecular event that became fixed in the most recent common ancestor of the MTBC after the phylogenetic separation from the M. canettii clade (Fig. 6). Indeed, while M. canettii strains possess a twin pks5 and pap conformation and synthesize LOS, all members of the classical

266 MTBC contain an abridged pks5 locus missing a full length pap gene and do not produce

267 LOS. Identification of a similar recombination event in a spontaneous R-variant of M. canettii

268 K reinforces the likelihood of this evolutionary scenario. Comparable recombination-deletion events also occurred for other genes, e.g. pknH (Supplementary Note).

Pks5 is a type-I polyketide synthase and a member of the broader polyketide-synthase

271 family, which in mycobacteria have important functions that range from catalyzing the 272 essential last condensation step of mycolic acid biosynthesis $(\mathrm{Pks} 13)^{38}$ to the synthesis of the 
273 polyketide backbone of phenolic glycolipids $(\mathrm{Pks} 15 / 1)^{39}$ or the synthesis of mycoketides $274(\mathrm{Pks} 12)^{40}$. In M. marinum, the two Pks5 polyketide synthases (Pks5 and Pks5.1) are thought 275 to be involved in the synthesis of polymethyl-branched fatty acids, which are further modified 276 by glycosyltransferases and methyltransferases encoded by genes adjacent to the pks5 277 genes $^{22,23,41}$ to produce LOS. Indeed, disruption of pks5 (MMAR_2340), analogous to 278 disruptions of the adjacent genes fadD25 (MMAR_2341), papA4 (MMAR_2343), and papA3 279 (MMAR_2355) leads to a complete loss of LOS, suggesting that besides the core pks5-pap 280 locus, several flanking genes are also involved in the early steps of LOS281 biosynthesis ${ }^{14,18,19,22,23,41,42}$ (Supplementary Table 3).

282 Within the group of tubercle bacilli, the overall genetic organization of the 283 orthologous LOS locus is similar between $M$. canettii and the non-functional version in $M$. 284 tuberculosis. However, apart from the recombined pks5 and the deleted pap gene, several 285 flanking genes show SNPs and small indels (Supplementary Table 4), some of which might 286 represent loss-of-function mutations involved in the non-complementation phenotype with 287 cosmid C9 in MTBC members. These findings suggest that after the phylogenetic separation 288 of the MTBC from the M. canettii-like progenitor pool, in addition to $p k s 5$ recombination and 289 pap deletion, other mutations in genes of the LOS locus accumulated in the MTBC in the 290 absence of selective pressure for sustaining LOS biosynthesis. While LOS production might 291 play an important role in generalist mycobacteria, it appears that its absence might provide a 292 selective advantage for specialized pathogens in the mammalian host.

293 This conclusion is supported by our different infection experiments, where loss of 294 LOS by M. canettii R-variants led to increased virulence in human macrophages and under in 295 vivo conditions, particularly for the sensitive guinea pig infection model. These data support a 296 scenario wherein the recombination of pks 5 genes in a common ancestor of the MTBC has 297 contributed to the evolutionary success and host adaptation of the resulting LOS-deprived 298 strains. This finding is in agreement with data for other mycobacterial species, where 299 increased virulence of R-morphotypes relative to S-morphotypes has been reported ${ }^{11,12,14}$, 300 although the involved glycolipids are different. In M. avium, for example, homologous 301 recombination within the ser 2 gene cluster likely led to phenotype changes linked to loss of 302 GPLs $^{43}$. For M. abscessus, a masking effect of GPLs on the outermost layer of the cell wall 303 of the S-morphotype strains has been suggested, which was related to repression of TLR2 304 responses $^{34}$. Similarly, LOS is located in the outermost layer of the mycobacterial cell 305 envelope $^{44}$ and thus might be interacting with other cell wall-associated 306 lipids/glycoconjugates such as phthiocerol dimycocerosates, lipoarabinomannans or the 19- 
$307 \mathrm{kD}$ lipoprotein ${ }^{45}$, which are important for host-pathogen interaction ${ }^{46}$. At present, it is difficult

308 to speculate by which mechanisms R-variants of tubercle bacilli might have gained a fitness 309 advantage during infection. However, differences we show here in TLR2-mediated 310 inflammatory responses between S- and R-variants of $M$. canettii $\mathrm{K}$ suggest that bacterial 311 surface remodelling could well have played an important role in the ancestor of the MTBC 312 during adaptation to mammalian hosts. In this respect, the proposed recombination of $p k s 5$ 313 and deletion of pap in a common ancestor of the MTBC adds to previously described 314 differences between $M$. canettii and the MTBC, such as the putative loss of vitamin B12 315 production due to the $c o b F$ deletion ${ }^{3,7,9,47,48}$, the integration of gene pe pgrs33 $(r v 1818 c)^{3}$, the 316 molecular scars in $p k s 8 / 17$ and three other gene pairs ${ }^{3,7,9}$, or the exchange of the exotoxin317 encoding domain in CpnT (Rv3903) $)^{9,49}$ (Fig. 6). While these reported differences are mainly 318 based on hypotheses formulated during comparative genomics and genome analysis 319 approaches, the virulence differences observed for rough $M$. canettii relative to isogenic LOS320 producing smooth $M$. canettii strains now provide first experimental support of our postulated 321 evolutionary model (Fig. 6). Recombination of two ancestral pks5 genes and consequent 322 remodelling of the bacterial cell surface thus represent key events in the emergence of the 323 professional pandemic pathogen M. tuberculosis.

\section{Authors to whom correspondence and requests for materials should be addressed:}

325 Roland Brosch (roland.brosch@pasteur.fr) or Christophe Guilhot

326 (Christophe.Guilhot@ipbs.fr)

\section{Acknowledgements}

328 We thank Torsten Seemann for initial help with NeighborNet analysis, and Hannes Pouseele 329 for help with mapping and SNP analysis. We are also grateful to Ida Rosenkrands and 330 Giovani Delogu for kindly providing polyclonal anti-SigA antibodies and vector pMV10-25, 331 respectively, and Karim Sébastien for expert assistance in animal care in biosafety-A3 332 facilities. We acknowledge the support by the European Community's grant 260872, the EU333 EFPIA Innovative Medicines Initiative (grant 115337), the Agence National de Recherche 334 (ANR-14-JAMR-001-02) and the Fondation pour la Recherche Médicale FRM 335 (DEQ20090515399 and DEQ20130326471). High-throughput sequencing was performed on 336 the Genomics Platform, member of the "France Génomique" consortium (ANR10-INBS-09-

337 08). R.B. is a member of the LabEx consortium IBEID at the Institut Pasteur. F. L-C was 338 supported by the French Region Ile-de- France (Domaine d'Intérêt Majeur Maladies 
339 Infectieuses et Emergentes) $\mathrm{PhD}$ program. E.C.B. was supported by a stipend from the 340 Pasteur-Paris University (PPU) International PhD program and the Institut Carnot Pasteur 341 Maladies Infectieuses.

\section{Author contributions}

344 E.C.B., C.G., L.M. and R.B. designed the study. E.C.B., W.F., F.LC., and A.P. performed

345 mycobacterial phenotypic assays and/or infection experiments. E.C.B., A.C. and R.B.

346 established genetic constructs. W.M., G.E., F.L., M.D. and C. G. generated and/or analysed 347 mycobacterial lipid and lipooligosaccharide profiles. E.C.B., L.Ma, C.B., M.O., T.P.S. and 348 P.S. generated and/or analysed sequence data. E.C.B and L.Majlessi conducted and analysed 349 immune assays. E.C.B., T.P.S., P.S., C.G. and R.B wrote the manuscript, with comments 350 from all authors.

\section{Competing financial interests}

353 P.S. is a consultant for Genoscreen. All other authors declare no competing financial interests.

\section{Accession codes}

356 The WGS-generated reads of the M. canettii $\mathrm{I}_{\mathrm{S} / \mathrm{R}}$ and $\mathrm{K}_{\mathrm{S} / \mathrm{R}}$ strains were deposited in the 357 European Nucleotide Archive (ENA) under accession number PRJEB11645 (ERP013045).

\section{Figure legends}

Fig. 1. Comparison of pks 5 genomic region of various mycobacterial strains. (A) pks 5 genetic region of M. kansasii ATCC 12478, M. marinum M, M. canettii A and M. tuberculosis H37Rv. Percentages on gray lines represent identity values as defined by ClustalW2 between genes of M. kansasii, and M. marinum or M. canettii A and M. marinum or M. tuberculosis H37Rv. The red arrows indicate location of primers used for long range PCR of pks 5 gene(s) in M. canettii strains I and K, as well as in M. tuberculosis H37Rv. (B) Results of long range PCR using above-mentioned primers that bind outside the pks 5 genes. M. tuberculosis H37Rv $(M t b) ; M$. canettii strains: $\mathrm{I}_{\mathrm{S}}, \mathrm{I}_{\mathrm{R}}, \mathrm{K}_{\mathrm{S}}$ and $\mathrm{K}_{\mathrm{R}}$. Note that for strains $\mathrm{I}_{\mathrm{S}}$ and $\mathrm{I}_{\mathrm{R}}$ unspecific fragments at $\sim 12 \mathrm{~kb}$ are visible. Data are representative of at least three repetitions. (C)

371 Genetic locus of pks5 genes in $M$. canettii strains $\mathrm{K}_{\mathrm{S}}$ and $\mathrm{K}_{\mathrm{R}}$ and potential recombination

372 region in $\mathrm{K}_{\mathrm{R}}$ (depicted by black arrows). Percentages on gray lines represent identity values as 373 defined by ClustalW2. (D) Domain organization of the pks5 genes of $M$. canettii strains $\mathrm{K}_{\mathrm{S}}$ 
and $\mathrm{K}_{\mathrm{R}}$. Domains were predicted according to the organization of pks 5 of $M$. tuberculosis

$375 \mathrm{H} 37 \mathrm{Rv}^{50}$ and their respective amino acid positions (AA) are depicted either above or below the particular domains. Sequences of individual domains of $p k s 5-1$ and $p k s 5-2$ were aligned using ClustalW2 and identity values are shown as percentage. Origin of the domains of the recombined pks5 of $M$. canettii strain $\mathrm{K}_{\mathrm{R}}$ is represented in different nuances of gray (light gray originating from $p k s 5-2$ and dark gray from $p k s 5-1$; medium gray indicates that domains are identical between $p k s 5-1$ and $p k s 5-2)$. Domain abbreviations: ketosynthase (KS), acyltransferase (AT), dehydratase (DH), enoylreductase (ER), ketoreductase (KR) and acylcarrier protein $(\mathrm{ACP})$.

Figure 2. Complementation of morphology phenotypes of $R$ variants. (A) Colony morphologies of $M$. canettii strains $\mathrm{K}_{\mathrm{S}}$ and $\mathrm{I}_{\mathrm{S}}$ and their rough mutants $\mathrm{K}_{\mathrm{R}}$ and $\mathrm{I}_{\mathrm{R}}$ complemented with the whole pks5 locus (C9) as well as M. tuberculosis H37Rv WT and $M$. tuberculosis::C9. Data are representative of at least ten repetitions (plating). Scale bar $=2.5$ mm. (B) M. canettii strains $\mathrm{K}_{\mathrm{S}}, \mathrm{K}_{\mathrm{R}}$ and $\mathrm{K}_{\mathrm{R}}:$ :C9 grown in $7 \mathrm{H} 9$ medium supplemented with ADC. Data are representative of at least ten repetitions. Scale bar $=5.0 \mathrm{~mm}$. (C) ZiehlNeelsen staining of $M$. canettii strains $\mathrm{K}_{\mathrm{S}}, \mathrm{K}_{\mathrm{R}}$ and $\mathrm{K}_{\mathrm{R}}:$ :C9. Data are representative of 2 repetitions. Scale bar $=10 \mu \mathrm{m}$.

Figure 3. PFGE and Southern hybridization analyses with PCR-derived probes binding either in a conserved domain of pks5 (middle panel) or within the pap gene (right panel). Genomes of strains were digested with $\mathrm{MfeI}$, resulting in either a 20 or a $12 \mathrm{~kb}$ fragment (black arrows). For all C9-complemented strains, the probe hybridized twice, with fragments of $20 \mathrm{~kb}$ and 12 $\mathrm{kb}$ confirming the presence of the natural and the vector-integrated $p k s 5$ loci. PFGE gels were run for $16 \mathrm{~h}$ with a pulse of $1 \mathrm{~s}$; M. canettii A; M. tuberculosis H37Rv (Mtb); M. canettii strains $\mathrm{K}_{\mathrm{S}}, \mathrm{K}_{\mathrm{R}}, \mathrm{K}_{\mathrm{R}}:$ :C9; $\mathrm{K}_{\mathrm{R}}$ ::vector-control; M. tuberculosis H37Rv::C9; cosmid C9; M: lowrange PFG Marker (NEB). Data are representative of three repetitions.

Figure 4. Deficient LOS production in rough morphotypes. (A) TLC analysis of lipid extracts from various M. canettii strains and M. tuberculosis H37Rv. Lipid extracts were dissolved in $\mathrm{CHCl}_{3}$ and run in $\left(\mathrm{CHCl}_{3}: \mathrm{CH}_{3} \mathrm{OH}: \mathrm{H}_{2} \mathrm{O}, 60: 24: 2\right)$. Glycolipids were visualized by spraying with anthrone, followed by charring. Insert corresponds to an enhanced contrast picture showing a faint blue spot corresponding to LOS in $M$. canettii A. This experiment was 
from $M$. canettii strains $\mathrm{I}_{\mathrm{S}}$ and $\mathrm{K}_{\mathrm{S}}$. Note that the mass peak distribution for the LOS 409 component of strain I was 14 mass-units lower than that observed for $\mathrm{K}$ and $\mathrm{A}$. Further 410 MS/MS fragmentation analysis showed successive losses of the O-methyl acylated trehalose 411 and oligosaccharides from the 3 major precursor-ions for strain I, and thus suggests that the 412 difference of 14 mass-units resulted from the presence of a rhamnosyl unit in LOS of strain I, 413 instead of a 2-O-Me-rhamnosyl residue linked to the $\beta$-glucosyl unit (L-Rhap- $\alpha 1->3-D-G l c p-$ $414 \beta 1->3)$ present in LOS of strains K. (C) TLC analysis $\left(\mathrm{CHCl}_{3}: \mathrm{CH}_{3} \mathrm{OH}: \mathrm{H}_{2} \mathrm{O}, 60: 24: 2\right)$ of lipid 415 extracts of $M$. canettii strains $\mathrm{I}_{\mathrm{S}}, \mathrm{I}_{\mathrm{R}}, \mathrm{I}_{\mathrm{R}}::$ vector-control, $\mathrm{I}_{\mathrm{R}}:: C 9$, as well as $\mathrm{K}_{\mathrm{S}}, \mathrm{K}_{\mathrm{R}}, \mathrm{K}_{\mathrm{R}}::$ vector 416 control, $\mathrm{K}_{\mathrm{R}}:: \mathrm{C} 9$ and M. tuberculosis H37Rv::C9.. This experiment is representative of two 417 experiments performed independently. $\mathrm{LOS}=$ lipooligosaccharide. (D) Structure of 418 lipooligosaccharides (LOS) from M. canettii. The acyl substituents, primarily 2L-,4L419 dimethylhexadecanoate, 2L-,4L-,6L-,8L-tetramethyloctadecanoate, and 2-methyl-3hydroxyeicosanoate, are at positions $2,3,6$ and 3,4,6 of the terminal glucosyl unit ${ }^{29}$.

Figure 5. Survival of morphotypes in different infection models. (A-B) Intracellular growth of M. tuberculosis $\mathrm{H} 37 \mathrm{Rv}, M$. canettii strains $\mathrm{K}_{\mathrm{S}}, \mathrm{K}_{\mathrm{R}}, \mathrm{K}_{\mathrm{R}}:: \mathrm{C} 9$ and $\mathrm{K}_{\mathrm{R}}:$ :vector-control in differentiated THP-1 macrophages. THP-1 cells were infected with various strains at an MOI of 0.05 ( $\sim 1$ bacterium per 20 cells). CFU of intracellular bacteria were determined $3 \mathrm{~h}$ and 3 , 4 and 5 days post infection (pi). The figure shows CFU numbers (A) and fold growth rates (B). Data are represented as means and standard deviation of at least four independent experiments. Significance in difference was determined using Two-way ANOVA $\left({ }^{*} p<0.05\right.$, $* * p<0.001, * * * p<0.0001$ ). (C) Survival of SCID mice infected with different morphotypes. $1 \times 10^{6} \mathrm{CFU}$ per mouse of $M$. tuberculosis $\mathrm{H} 37 \mathrm{Rv}$, M. canettii strains $\mathrm{K}_{\mathrm{S}}, \mathrm{K}_{\mathrm{R}}$ or $\mathrm{K}_{\mathrm{R}}:: \mathrm{C} 9$ were IV-injected and survival of mice was monitored. Endpoints were defined as loss of $>20 \%$ of 432 body weight. The initial dose of all strains was comparable with $4.6 \times 10^{6} \mathrm{CFU} / \mathrm{ml}$ for $433 \mathrm{H} 37 \mathrm{Rv}, 3.6 \times 10^{6} \mathrm{CFU} / \mathrm{ml}$ for $\mathrm{K}_{\mathrm{S}}, 4.1 \times 10^{6} \mathrm{CFU} / \mathrm{ml}$ for $\mathrm{K}_{\mathrm{R}}$ and $3.3 \times 10^{6} \mathrm{CFU} / \mathrm{ml}$ for $\mathrm{K}_{\mathrm{R}}:: \mathrm{C} 9$. 434 Data represent one experiment with 10 mice per group. (D) Bacterial load of morphotypes in lungs of infected guinea pigs. Guinea pigs were infected via aerosol route with $2.5 \times 10^{6} \mathrm{CFU}$. Lungs of infected animals were homogenized at day 1 and day 42 pi and CFU of different morphotypes were determined. Data represent median with interquartile range of CFU at day 43842 divided by CFU at day 1 of two biological replicates with 4 guinea pigs per group (strains $439 \mathrm{~K}_{\mathrm{S}}$ and $\left.\mathrm{K}_{\mathrm{R}}\right)$ or 1 experiment with 4 guinea pigs per group $\left(\mathrm{K}_{\mathrm{R}}: \mathrm{C} 9\right)$. Significant difference was 440 observed using Kruskal-Wallis test with Dunn's correction $\left({ }^{*} p<0.05,{ }^{* *} p<0.01\right)$. (E-F) IL-6 and IL-12p40 production in BM-DCs upon infection with different morphotypes. BM-DCs of 
442 C57BL/6 WT were infected with Sauton-grown M. tuberculosis H37Rv, M. canettii strains $443 \mathrm{~K}_{\mathrm{S}}, \mathrm{K}_{\mathrm{R}}$ or $\mathrm{K}_{\mathrm{R}}: \mathrm{C} 9$ at an MOI of $1.24 \mathrm{~h}$ pi, levels of IL-6 and IL-12p40 in the cell supernatants 444 were determined by ELISA. Note that a dominant inhibitory effect of LOS on the induction of 445 inflammatory responses was excluded since co-infection of BM-DCs with strains $\mathrm{K}_{\mathrm{S}}$ and $\mathrm{K}_{\mathrm{R}}$ 446 did not result in less cytokine production than infection with $M$. canettii $\mathrm{K}_{\mathrm{R}}$ alone. Data are 447 represented as means and standard deviation of three independent experiments. Significance 448 in difference was determined using Mann Whitney test $\left({ }^{*} p<0.05\right)$. (G-H) Differential 449 interaction of S- and R-morphotypes with TLR2. Human TLR2- or TLR4-transfected 450 HEK293 cells were incubated with S- and R-morphotypes at an MOI of 1. At $24 \mathrm{~h}$ pi, levels 451 of secreted embryonic alkaline phosphatase reporter gene under the control of NF- $\kappa \mathrm{B}$ were 452 measured with a spectrophotometer. PAM3CSK4 $(10 \mu \mathrm{g} / \mathrm{ml})$ and LPS $(100 \mathrm{ng} / \mathrm{ml})$ were used 453 as positive controls for TLR2- or TLR4-mediated stimulation, respectively. Data are 454 represented as means and standard deviation of at least three independent experiments. 455 Significance in difference was determined using Mann-Whitney test $(* * * p<0.001)$. n.s. non456 stimulated.

458 Figure 6. Scheme showing supposed molecular key events in mycobacterial evolution from 459 the recombinogenic $M$. canettii strain pool of putative environmental origin, towards 460 professional pathogens of mammalian hosts evolved by clonal expansion of one emerging 461 sublineage. Network phylogeny inferred among eight $M$. canettii strains used in this study, 462 and $46 \mathrm{MTBC}$ strains by NeighborNet analysis based on genome sequence data. Gene names 463 and arrow shown in grey refer to previously described differences between $M$. canettii strains 464 and MTBC members ${ }^{9}$, whereas the pks5 recombination event is marked by a red arrow. 465 Figure adapted from Bottai et al. ${ }^{1}$ and Boritsch et al. ${ }^{9}$. 
Mycobacterial strains and growth conditions

470 Cloning was performed in LB-grown Escherichia coli XL-2 (Stratagene) using hygromycin

$471\left(200 \mu \mathrm{g} \cdot \mathrm{ml}^{-1}\right)$ or kanamycin $\left(50 \mu \mathrm{g} \cdot \mathrm{ml}^{-1}\right)$ selection. MTBC and M. canettii strains were 472 grown in Middlebrook 7H9 broth (Becton-Dickinson) containing albumin-dextrose-catalase 473 (ADC) or on Middlebrook 7H11 medium (Becton-Dickinson) containing oleic acid-albumin474 dextrose-catalase (OADC) or in Sauton medium (when specified) at $37^{\circ} \mathrm{C}$. Hygromycin (50 $475 \mu \mathrm{g} \cdot \mathrm{ml}^{-1}$ ) was used for mycobacterial selection.

\section{Genome analysis of $S / R$ morphotype isolates}

Mycobacterial DNA was prepared by standard procedures ${ }^{16,17}$. After final ethanol precipitation and washing, DNA was resuspended in TE buffer and used for library preparation and Illumina-based genome sequencing. This approach yielded on average 21 million $\left(I_{S}\right.$ and $\left.I_{R}\right)$ and 8 million $\left(K_{S}\right.$ and $\left.K_{R}\right)$ reads per strain, which were then mapped against the corresponding reference sequences of STB-I (ENA WGS project CAOO00000000) and STB-K (NC_019951). Reads were aligned against the reference genomes $^{3}$ using SHRiMP ${ }^{51}$. Alignment maps were visualized with Tablet ${ }^{52}$ and SNPs were called according to coverage sums and variant frequencies. At least 10 reads had to be matched with a substitution frequency $>0.89$ to guarantee the detection of high confidence SNPs. Additional mapping and SNP analysis was performed using BioNumerics v7.6 software (Applied Maths), using parameters pre-calibrated based on re-sequencing data of reference genomes ${ }^{53}$.

\section{Network phylogenic analysis of $M$. canettii and MTBC strains}

492 Network phylogeny among $M$. canettii and MTBC strains was inferred by NeighborNet 493 analysis, based on pairwise alignments of whole-genome SNP data. Phylogenetic groupings were identified by split decomposition analysis using the SplitsTree4 software ${ }^{54}$.

\section{Long-range PCR and pks5-sequencing}

pks5 and/or pap genes were amplified from chromosomal DNA using Platinum-Taq polymerase (Life Technologies) according to the manufacturer's manual with primers EB-1 TTTATTAATCAGGGAAAGGCGACATCGGA and EB-2 TTTTTATAACCGCCAAGAC AAACTTCATC for annealing at $55^{\circ} \mathrm{C}$ and elongation at $68^{\circ} \mathrm{C}(10 \mathrm{~min})$. Amplicons were

501 Sanger-sequenced after QIAquick (Quiagen) purification, using primers listed in 
502 supplementary Table S5. Amplification of $p k s 5-1$ with the last part of pap or amplification of 503 pks5-2 with the first part of pap to verify the presence of both pks5 genes in the various $M$. 504 canettii strains was performed using Pwo Polymerase (Roche) and the oligos

TTTCGCTACCAACGACTAGTAGTTCGTC, respectively.

\section{Complementation of R-variants}

511 R-variants of M. canettii strains K and I, as well as M. tuberculosis H37Rv and other MTBC members were transformed with the integrating cosmid C9 and H6 spanning the extended or partial pks5 locus of $M$. canettii strain A. The C9 and H6 clones were selected from a cosmid

514 library that was constructed in pYUB $412^{28}$ by using partially-digested, agarose-embedded genomic DNA ${ }^{55,56}$ of $M$. canettii reference strain CIPT 140010059 (STB-A). Clone-inserts were PCR-verified using primers binding in vector- or insert-sequences: (T7-F AGGCATGCAAGCTCAGGATA; T7-R GGATCGGTCCAGTAATCGT and T3-F GCAGAAGCACTAGACGATCC; T3-R GCCGCAATTAACCCTCACTA). Transformed, hygromycin-resistant R-variants that exhibited no S-morphology served as vector-controls. Insert-termini sequencing of these latter clones showed partial insert-loss including the $p k s 5$ region, whereas genes encoding MCAN_15411, MCAN_15421 and part of MCAN 15431 remained present.

For construction of vectors expressing HA-tagged proteins, corresponding genes were amplified with Pwo polymerase (Roche). For the construction of the HA-tagged pks5-2 expression plasmid, pTTP $1 b^{57}$ was cut with HindIII (NEB) to remove the kanamycin resistance cassette and blunt ends were generated using Klenow fragment (NEB). A

527 hygromycin resistance cassette was amplified from pAL70 ${ }^{58}$ with the oligos AC123 ACAGGCCTGGATGCCAGGGCCTTTCA, cut with StuI and cloned into the digested vector to generate pAL232. The strong mycobacterial promoter of the gene hsp60 was amplified

531 from chromosomal DNA of M. tuberculosis H37Rv with the oligos NheI_HindII_hsp60_F aaaGCTAGCAAGCTTggtgaccacaacgacgcgeccgetttgatc and hsp60_SpeI_EcoRV_XbaI_R aaaTCTAGAgatatcACTAGTtgtcttggccattgcgaagtgattcctcc, the amplified product was cut with NheI and XbaI (NEB) and cloned into XbaI-digested pAL232 to generate pEB18. The gene pks5-2 was amplified from chromosomal DNA of $M$. canettii strain $\mathrm{K}_{\mathrm{S}}$ with the oligos 
pks5-2_SpeI_F aaaACTAGTGTGGGTAAGGAGAGAACAAAG and pks5-2_HA_PsiI_R aaTTATAAttaAGCATAATCAGGAACATCATACGGATATGAAGGTGCTGCAATGTC

538 GG. The amplified product was cut with SpeI and Psi I and cloned into SpeI-EcoRV digested pEB18. For construction of the HA-tagged pap expression system, the plasmid pMV10-25 was first cut with NcoI, blunt ended using Klenow fragment (NEB) and subsequently cut with NheI. The pap gene was amplified using the oligos pap_SpeI_F aaACTAGTGTGATCATTGGCGGGGGC and pap_HA_PsiI_R aaTTATAAttaAGCATAATCAGGAACATCATACGGATAGCTAGATACGCGAACTGC TG. The amplified product was digested with SpeI and Psi and cloned into the NheI/ blunt end pMV10-25 ${ }^{59}$ vector.

546 Whole cell lysates were generated from cultures grown in Middelbrook $7 \mathrm{H} 9$ supplemented with ADC to an OD of 0.6-0.8. Cells were disrupted using a TissueLyser II (Quiagen) and Zirkonia/ Silica beads (BioSpec) and passed through $0.2 \mu \mathrm{m}$ filter units. Proteins were separated on NuPAGE Novex 10\% Bis-Tris gels (Invitrogen) for HA-tagged pap and NuPAGE Novex 4-12\% Bis-Tris gels (Invitrogen) for HA-tagged pks5-2. HA-tagged pap was blotted on a nitrocellulose membrane using an iBlot Dry Blotting System

552 (LifeTechnologies), while pks5-2 was blotted on nitrocellulose membranes using a wet transfer system for $2 \mathrm{~h}$ at $100 \mathrm{~V}$ at $4^{\circ} \mathrm{C}$. Membranes were blocked in 5\% milk powder in TBS and subsequently incubated with either mouse anti-HA (anti-HA.11; Covance) or rabbit anti-

555 SigA (Statens Serum Institute, Copenhagen, Denmark) primary antibody. Bound antibodies

556 were detected using a horseradish peroxidase-conjugated sheep anti-mouse or donkey anti557 rabbit (Amersham ECL) antibody and the chemiluminescent signal was developed with a 558 SuperSignal West Femto Maximum Sensitivity Substrate (Thermo Scientific). Image 559 acquisition was performed with an Azure c300 (Azure Biosystems).

\section{Ziehl-Neelsen staining}

562 Mycobacteria were grown to an OD of 0.4 , smeared on glass slides and killed at $95^{\circ} \mathrm{C}(1 \mathrm{~h})$. 563 After heat-fixation with 90\% ethanol, mycobacteria were stained with carbol-fuchsin and 564 heated (10 $\mathrm{min})$. After washing, glass slides were incubated with sulphuric acid (25\%) (2 $565 \mathrm{~min}$ ), washed and incubated with $90 \%$ ethanol (5 min), washed and counter-stained with 566 methylene blue (2 $\mathrm{min})$.

\section{PFGE and Southern Blot hybridization}


569 Genomic DNA in agarose-plugs, prepared as described ${ }^{55}$, was MfeI-digested, separated by 570 pulsed-field gel electrophoresis (Biorad CHEF II, pulse $1 \mathrm{~s}$ for $16 \mathrm{~h}$ at $6 \mathrm{~V} \cdot \mathrm{cm}^{-1}$ ) and 571 transferred onto Hybond-C-Extra nitrocellulose (GE), as described ${ }^{55}$. Hybridization was 572 performed with $\left[\alpha-{ }^{32} \mathrm{P}\right] \mathrm{dCTP}-$ labeled PCR-probes at $68^{\circ} \mathrm{C}$ in $6 \mathrm{xSSC} / 0,5 \% \mathrm{SDS} / 0.01 \mathrm{M}$ 573 EDTA/ 5xDenhardt's solution/ $100 \mathrm{mg} \cdot \mathrm{ml}^{-1}$ salmon-sperm DNA. After washing, membranes 574 were exposed to phosphorimager screens, which were scanned in a STORM phosphorimager. 575 Probes were amplified from M. tuberculosis DNA using pks5 primers (pks5-F 576 GTTGTGGGAGGCGTTGCT; pks5-R GAAACGTCGAACGCATGAC) or from $M$. canettii $577 \mathrm{~K}_{\mathrm{S}}$ using pap primers (pap-F CTCGATTATTCACGGCTGGT; pap-R 578 CGTATAGCCCGGTGATCAAC).

$580 \quad$ Extraction and analysis of lipooligosaccharides

581 Mycobacterial cells obtained from 7H9-grown cultures were exposed to $\mathrm{CHCl}_{3} / \mathrm{CH}_{3} \mathrm{OH}(1: 2$, $582 \mathrm{v} / \mathrm{v}$ ) for $48 \mathrm{~h}$ to kill bacteria. Lipids were extracted following previously described 583 protocols ${ }^{14,21}$ and separated by TLC. Finally, glycolipids were visualized by spraying the 584 plates with a $0.2 \%$ anthrone solution $(\mathrm{w} / \mathrm{v})$ in concentrated $\mathrm{H}_{2} \mathrm{SO}_{4}$, followed by heating. The previously described LOS of $M$. canettii strain CIPT 140010059 was used as standard ${ }^{29}$. 586 Crude lipid extracts were subjected to chromatography on a Sep-Pak Florisil cartridge and 587 eluted at various concentrations of $\mathrm{CH}_{3} \mathrm{OH}(0,5,10,15,20,30 \%)$ in $\mathrm{CHCl}_{3}$. Each fraction was analyzed by TLC on Silica Gel G60 using $\mathrm{CHCl}_{3} / \mathrm{CH}_{3} \mathrm{OH} / \mathrm{H}_{2} \mathrm{O}(30: 12: 1$, v/v/v) as solvent and glycolipids were visualized as described above.

MALDI-TOF/TOF-MS and MS/MS analyses were conducted in the positive ionization and reflectron mode by accumulating 10 spectra of 250 laser shots, using the 5800 MALDI TOF/TOF Analyser (Applied Biosystems/Absciex) equipped with a Nd:Yag laser (349nm). For MS and MS/MS data acquisitions, uniform, continuous, and random stage motion was selected at a fixed laser intensity of 4000 (instrument-specific units) and $400 \mathrm{~Hz}$ pulse rate and 6000 (instrument-specific units) and $1000 \mathrm{~Hz}$, respectively. For MS/MS data acquisition, the fragmentation of selected precursor-ions was performed at collision energy of $1 \mathrm{kV}$. Lipid samples were dissolved in chloroform and spotted onto the target plate as $0.5 \mu 1$ droplets, followed by the addition of $0.5 \mu 1$ matrix solution (10 $\mathrm{mg}$ of 2,5-dihydroxybenzoic acid [Sigma-Aldrich] $/ \mathrm{ml}$ in $\mathrm{CHCl}_{3} / \mathrm{CH}_{3} \mathrm{OH}, 1 / 1$ [vol/vol]. Samples were allowed to crystallize at room temperature. Spectra were externally calibrated using lipid standards. strains was performed with $1 \mathrm{M}$ sodium methanolate for $1 \mathrm{~h}$ at $37^{\circ} \mathrm{C}$. After neutralization with 
603 glacial acetic acid, the mixture was dried under stream of nitrogen and lipids were extracted 604 with diethyl ether and washed twice with water. The resulting fatty acid methyl esters were 605 converted to trimethylsilyl (TMS) derivatives, using a mixture of 606 pyridine/hexamethyldisilazane/trimethylchlorosilane $(6: 4: 2, \mathrm{v} / \mathrm{v} / \mathrm{v})$.

607 GC-MS analyses were performed using a Thermo TraceGCultra chromatograph 608 coupled with an ISQ mass spectrometer. Chromatographic separations of the TMS derivatives 609 of the fatty acid methyl esters were obtained using an Inferno ZB5HT column of $15 \mathrm{~m}$. Helium 610 was the carrier gas at constant flow rate of $1.2 \mathrm{~mL} \mathrm{~min}^{-1}$. The oven temperature program was 611 started at $120^{\circ} \mathrm{C}$, ramped to $380^{\circ} \mathrm{C}$ at $10^{\circ} \mathrm{C} / \mathrm{min}$ (with final isothermal step of $5 \mathrm{~min}$ at $380^{\circ} \mathrm{C}$ ). 612 The temperature of the injector was $220^{\circ} \mathrm{C}$ and injection of $1 \mu 1$ of samples in petroleum ether 613 was performed in a split mode (ratio of 20:1). EI mass spectra were recorded using electron energy of $70 \mathrm{eV}$ from 60 to 600 with a transfer line maintained at $275^{\circ} \mathrm{C}$.

To visualize various polyketide-derive lipids, including acyl-trehaloses such 616 diacyltrehaloses (DAT), polyacyltrehaloses (PAT), sulfolipids (SL), phenolic glycolipids 617 (PGL) or lipooligosaccharides (LOS) from different $M$. canettii strains metabolic labelling with ${ }^{14} \mathrm{C}$ propionate was used. For this TLC analysis, $M$. canettii strains were grown to the exponential phase in $10 \mathrm{~mL} 7 \mathrm{H} 9$ liquid medium supplemented with $\mathrm{ADC}$ and $0.05 \%$ Tween 80 and labelled by incubation with $0.4 \mu \mathrm{Ci} \cdot \mathrm{ml}^{-1}\left[1-{ }^{14} \mathrm{C}\right]$ propionate for $24 \mathrm{~h}$. The TLC plates were run in $\mathrm{CHCl}_{3} / \mathrm{CH}_{3} \mathrm{OH} / \mathrm{H}_{2} \mathrm{O}(60 / 16 / 2)$ for DAT and $\mathrm{SL}$ and $\mathrm{CHCl}_{3} / \mathrm{CH}_{3} \mathrm{OH}(99 / 1)$ for PAT. Labelled lipids were visualized with a Typhoon PhosphorImager (Amersham 623 Biosciences).

\section{Macrophage infections}

626 THP-1 (TIB-202D) human monocyte-like cells were purchased from ATCC, directly 627 amplified and stocked in liquid nitrogen. Only low passage cells (passage number $<11$ ) were 628 used in the experiments. The purchased THP-1 cell line has been authenticated and tested for 629 microbial contaminants, including mycoplasma, by ATCC. For the experiments, THP-1 were 630 cultivated in RPMI 1640, GlutaMAX (Life Technologies) containing $10 \%$ heat-inactivated 631 fetal bovine serum (Life Technologies), seeded at a density of $7.5 \times 10^{4}$ cells per well in 96 well plates and differentiated into macrophages through incubation with $50 \mathrm{mM}$ PMA for 3

633 days. For infection, bacteria grown in Sauton medium without shaking were sonicated, added 634 to the macrophages at an MOI of 0.05 , ( 1 bacterium per 20 THP-1 cells $)$ and incubated for 2 635 h. Sauton medium was used, as it allows the production of more complex polar lipids ${ }^{18}$. After 636 phagocytosis, $0.1 \mathrm{mg} \cdot \mathrm{ml}^{-1}$ amikacin was added for $1 \mathrm{~h}$ to remove extracellular bacteria and 
637 cells were incubated for up to 6 days at $37^{\circ} \mathrm{C}$ and $5 \% \mathrm{CO}_{2}$. At various time points macrophages were lysed with $0.1 \%$ Triton-X100 in PBS and lysates plated in serial dilutions on $7 \mathrm{H} 11+\mathrm{OADC}$ plates to determine intracellular survival of bacteria in CFU. Experiments were performed as at least four biological replicates, each done in triplicate (technical replicates).

Additional infection experiments were conducted using Raw murine macrophages as well as Human monocyte-derived macrophages (hMDMs). Raw cells were cultivated in RPMI 1640 Medium, GlutaMAX (Gibco, Life Technologies) supplemented with $5 \%$ heatinactivated fetal bovine serum (Gibco, Life Technologies) and seeded one day before infection. In parallel, hMDMs were obtained from buffy coats by centrifugation of blood from healthy human donors in lymphocyte separation medium (Eurobio) and further isolation of $\mathrm{CD} 4^{+}$monocytes from the mononuclear cell fraction using CD14 microbeads (Miltenyi Biotec). Monocytes were differentiated into macrophages in the presence of rhM-CSF (50 $\mathrm{ng} / \mathrm{ml}$; R\&D Systems). At the day of infection mycobacterial strains grown in Sauton medium without shaking were sonicated, added to the macrophages at a MOI of 1:20, and incubated for $2 \mathrm{hrs}$ at $37^{\circ} \mathrm{C}$ and $5 \% \mathrm{CO}_{2}$. After phagocytosis, infected macrophages were incubated with $0.1 \mathrm{mg} \cdot \mathrm{ml}^{-1}$ amikacin for $1 \mathrm{~h}$ to remove extracellular bacteria and finally incubated for 6 days in new RPMI supplemented with $10 \%$ FBS for hMDMs and $1 \%$ for Raw cells at $37^{\circ} \mathrm{C}$ and 5 $\% \mathrm{CO}_{2}$. At selected time points macrophages were lysed with $0.1 \%$ Triton-X100 in PBS and bacteria were plated in serial dilutions on $7 \mathrm{H} 11$ plates supplemented with OADC to determine CFU counts. Buffy coats were obtained from healthy donors after informed consent (Etablissement Français du Sang). Three biological replicates were performed, each done in triplicate (technical replicates).

\section{Animal infection studies}

662 Six-week-old female SCID mice (Charles River) were infected intravenously with $200 \mu 1$ of $6635 \times 10^{6}$ bacteria/mouse and survival of mice was monitored. Humane endpoints were defined 664 as loss of $>20 \%$ of body-weight. The experiment was performed once, using 10 mice per 665 strain (technical replicates). In a second well-established mycobacterial infection model, five to six week-old female guinea pigs (Hartley; Charles River) were aerosol-infected using $5 \mathrm{ml}$ of a suspension containing $5 \times 10^{5}$ bacteria $\cdot \mathrm{ml}^{-1}$. Six weeks post-infection, animals were killed and organs homogenized using a gentleMACS Dissociator (Miltenyi Biotec) and gentleMACS M tubes. Bacterial loads in organs were determined by plating serial dilutions of organ homogenates on solid medium. The number of animals included in the experiments 
671 (sample size choice) was determined by taking into account the rule of 3Rs (replacement, 672 reduction, refinement) and statistical requirements. As regards randomization and blinding,

673 CFU counts were recorded in parallel by different investigators.

674

675 Cytokine and chemokine assays and HEK-Blue cell reporter assay

676 ELISA and ProcartaPlex Luminex immunoassay from culture supernatants of infected BM-

677 DCs was performed as previously described ${ }^{60}$, and/or according to the manufacturer's 678 instructions (ProcartaPlex Luminex Immunoassay). mAbs specific to IL-12p40 and IL-6 were 679 from BD Biosciences. Reagents for quantitative ProcartaPlex Luminex immunoassay were 680 from affymetrix eBioscience. ELISA experiments were performed as three biological 681 replicates, while Luminex analyses were performed as two independent biological replicates, 682 each done in duplicate. Signal acquisition was performed on pooled duplicates.

683 HEK-TLR2 and HEK-TLR4 cells (InvivoGen) were grown in DMEM medium (Gibco Life 684 Technologies) supplemented with 10\% FBS, $100 \mu \mathrm{g} \cdot \mathrm{ml}^{-1}$ Normocin and 1X HEK-Blue 685 Selection. TLR stimulation assay was performed according to the manufacturer's protocol. 10 $\mu \mathrm{g} \cdot \mathrm{ml}^{-1}$ PAM3CSK4 served as positive control for HEK-TLR2 and $100 \mathrm{ng} \cdot \mathrm{ml}^{-1}$ LPS as positive control for HEK-TLR4. Experiments were performed as at least three biological replicates.

689

\section{Compliance with regulations on animal welfare and ethics}

691 Animal studies were performed in agreement with European and French guidelines (Directive 692 86/609/CEE and Decree 87-848 of 19 October 1987) after approval by the Institut Pasteur 693 Safety Committee (Protocol 11.245) and local ethical committees (CNREEA 2012-0061; 694 CETEA 2013-0036).

\section{References:}

6971 Bottai, D., Stinear, T. P., Supply, P. \& Brosch, R. Mycobacterial Pathogenomics and 698 Evolution Microbiology Spectrum 2, MGM2-0025-2013 (2014).

6992 Le Chevalier, F., Cascioferro, A., Majlessi, L., Herrmann, J. L. \& Brosch, R. $700 \quad$ Mycobacterium tuberculosis evolutionary pathogenesis and its putative impact on drug development. Future Microbiol. 9:969-85., (2014).

3 Supply, P. et al. Genomic analysis of smooth tubercle bacilli provides insights into ancestry and pathoadaptation of Mycobacterium tuberculosis. Nat Genet 45, 172-179 (2013).

4 van Soolingen, D. et al. A novel pathogenic taxon of the Mycobacterium tuberculosis complex, Canetti: characterization of an exceptional isolate from Africa. Int J Syst Bacteriol 47, 1236-1245. (1997). 
7085 Gutierrez, M. C. et al. Ancient origin and gene mosaicism of the progenitor of

$709 \quad$ Mycobacterium tuberculosis. PLoS Pathog. 1, e5.

$7106 \quad$ Koeck, J. L. et al. Clinical characteristics of the smooth tubercle bacilli 'Mycobacterium canettii' infection suggest the existence of an environmental reservoir. Clin Microbiol Infect 17, 1013-1019 (2011).

$7 \quad$ Blouin, Y. et al. Progenitor "Mycobacterium canettii" Clone Responsible for Lymph Node Tuberculosis Epidemic, Djibouti. Emerg Infect Dis 20, 21-28, (2014). Dormans, J. et al. Correlation of virulence, lung pathology, bacterial load and delayed type hypersensitivity responses after infection with different Mycobacterium tuberculosis genotypes in a BALB/c mouse model. Clin Exp Immunol. 137, 460-468. (2004).

9 Boritsch, E. C. et al. A glimpse into the past and predictions for the future: the molecular evolution of the tuberculosis agent. Mol Microbiol. 93, 835-852. (2014).

10 Panas, M. W. et al. Noncanonical SMC protein in Mycobacterium smegmatis restricts maintenance of Mycobacterium fortuitum plasmids. Proc Natl Acad Sci U S A. 111, 13264-13271. (2014).

11 Kansal, R. G., Gomez-Flores, R. \& Mehta, R. T. Change in colony morphology influences the virulence as well as the biochemical properties of the Mycobacterium avium complex. Microb Pathog 25, 203-214, (1998).

12 Catherinot, E. et al. Hypervirulence of a rough variant of the Mycobacterium abscessus type strain. Infect Immun 75, 1055-1058, (2007).

13 Belisle, J. T. \& Brennan, P. J. Chemical basis of rough and smooth variation in mycobacteria. J Bacteriol. 171, 3465-3470. (1989).

14 van der Woude, A. D. et al. Unexpected link between lipooligosaccharide biosynthesis and surface protein release in Mycobacterium marinum. J Biol Chem 287, $20417-$ 20429, (2012).

15 Barrow, W. W. \& Brennan, P. J. Isolation in high frequency of rough variants of Mycobacterium intracellulare lacking C-mycoside glycopeptidolipid antigens. $J$ Bacteriol. 150, 381-384. (1982).

16 Howard, S. T. et al. Spontaneous reversion of Mycobacterium abscessus from a smooth to a rough morphotype is associated with reduced expression of glycopeptidolipid and reacquisition of an invasive phenotype. Microbiology 152, 1581-1590, (2006).

17 Pawlik, A. et al. Identification and characterization of the genetic changes responsible for the characteristic smooth-to-rough morphotype alterations of clinically persistent Mycobacterium abscessus. Mol.Microbiol.90, 612-629. (2013).

18 Burguiere, A. et al. LosA, a key glycosyltransferase involved in the biosynthesis of a novel family of glycosylated acyltrehalose lipooligosaccharides from Mycobacterium marinum. J Biol Chem. 280, 42124-42133. (2005).

19 Ren, H. et al. Identification of the lipooligosaccharide biosynthetic gene cluster from Mycobacterium marinum. Mol Microbiol 63, 1345-1359 (2007).

20 Lemassu, A., Levy-Frebault, V. V., Laneelle, M. A. \& Daffe, M. Lack of correlation between colony morphology and lipooligosaccharide content in the Mycobacterium tuberculosis complex. J Gen Microbiol. 138, 1535-1541. (1992).

21 Etienne, G. et al. Identification of the polyketide synthase involved in the biosynthesis of the surface-exposed lipooligosaccharides in mycobacteria. J Bacteriol 191, 26132621 (2009).

22 Nataraj, V. et al. MKAN27435 is required for the biosynthesis of higher subclasses of lipooligosaccharides in Mycobacterium kansasii. PLoS One. 10, e0122804. (2015). 
23 Minnikin, D. E. et al. in Tuberculosis - Expanding Knowledge (ed W. Ribon) Ch. 7, (InTech, 2015).

24 Stinear, T. P. et al. Insights from the complete genome sequence of Mycobacterium marinum on the evolution of Mycobacterium tuberculosis. Genome Res 18, 729-741. (2008).

25 Wang, J. et al. Insights on the Emergence of Mycobacterium tuberculosis from the Analysis of Mycobacterium kansasii. Genome Biol Evol. 7, 856-870. doi: 810.1093/gbe/evv1035. (2015).

26 Quadri, L. E. Biosynthesis of mycobacterial lipids by polyketide synthases and beyond. Crit Rev Biochem Mol Biol. 49, 179-211. (2014).

27 Rousseau, C. et al. Virulence attenuation of two Mas-like polyketide synthase mutants of Mycobacterium tuberculosis. Microbiology. 149, 1837-1847. (2003).

28 Bange, F. C., Collins, F. M. \& Jacobs, W. R., Jr. Survival of mice infected with Mycobacterium smegmatis containing large DNA fragments from Mycobacterium tuberculosis. Tuber Lung Dis 79, 171-180. (1999).

29 Daffe, M., McNeil, M. \& Brennan, P. J. Novel type-specific lipooligosaccharides from Mycobacterium tuberculosis. Biochemistry. 30, 378-388. (1991).

30 Angala, S. K., Belardinelli, J. M., Huc-Claustre, E., Wheat, W. H. \& Jackson, M. The cell envelope glycoconjugates of Mycobacterium tuberculosis. Crit Rev Biochem Mol Biol. 49, 361-399. (2014).

31 Onwueme, K. C., Ferreras, J. A., Buglino, J., Lima, C. D. \& Quadri, L. E. Mycobacterial polyketide-associated proteins are acyltransferases: proof of principle with Mycobacterium tuberculosis PapA5. Proc Natl Acad Sci U S A. 101, 4608-4613. (2004).

32 Bhatt, K., Gurcha, S. S., Bhatt, A., Besra, G. S. \& Jacobs, W. R., Jr. Two polyketidesynthase-associated acyltransferases are required for sulfolipid biosynthesis in Mycobacterium tuberculosis. Microbiology. 153, 513-520. (2007).

33 Bottai, D. et al. Increased protective efficacy of recombinant BCG strains expressing virulence-neutral proteins of the ESX-1 secretion system. Vaccine. 33, 2710-2718. (2015).

34 Rhoades, E. R. et al. Mycobacterium abscessus Glycopeptidolipids mask underlying cell wall phosphatidyl-myo-inositol mannosides blocking induction of human macrophage TNF-alpha by preventing interaction with TLR2. J Immunol 183, 19972007, (2009).

35 Roux, A. L. et al. Overexpression of proinflammatory TLR-2-signalling lipoproteins in hypervirulent mycobacterial variants. Cell Microbiol 13, 692-704, (2011).

36 Robinson, R. T., Orme, I. M. \& Cooper, A. M. The onset of adaptive immunity in the mouse model of tuberculosis and the factors that compromise its expression. Immunol Rev. 264, 46-59. (2015).

37 Achtman, M. Insights from genomic comparisons of genetically monomorphic bacterial pathogens. Philos Trans R Soc Lond B Biol Sci 367, 860-867 (2012).

38 Portevin, D. et al. A polyketide synthase catalyzes the last condensation step of mycolic acid biosynthesis in mycobacteria and related organisms. Proc Natl Acad Sci US A 101, 314-319. (2004).

39 Constant, P. et al. Role of the pks $15 / 1$ gene in the biosynthesis of phenolglycolipids in the Mycobacterium tuberculosis complex. Evidence that all strains synthesize glycosylated $p$-hydroxybenzoic methyl esters and that strains devoid of phenolglycolipids harbor a frameshift mutation in the $p k s 15 / 1$ gene. J Biol Chem 277, 38148-38158, (2002). 
40 Matsunaga, I. et al. Mycobacterium tuberculosis pks 12 produces a novel polyketide presented by CD1c to T cells. J Exp Med. 200, 1559-1569. (2004).

41 Rombouts, Y. et al. Fatty acyl chains of Mycobacterium marinum lipooligosaccharides: structure, localization and acylation by PapA4 (MMAR_2343) protein. J Biol Chem. 286, 33678-33688. (2011)

43 Eckstein, T. M., Inamine, J. M., Lambert, M. L. \& Belisle, J. T. A genetic mechanism for deletion of the ser 2 gene cluster and formation of rough morphological variants of Mycobacterium avium. J Bacteriol 182, 6177-6182 (2000).

44 Ortalo-Magne, A. et al. Identification of the surface-exposed lipids on the cell envelopes of Mycobacterium tuberculosis and other mycobacterial species. $J$ Bacteriol 178, 456-461 (1996).

45 Mortaz, E. et al. Interaction of Pattern Recognition Receptors with Mycobacterium tuberculosis. J Clin Immunol 14, 14 (2014).

46 Cambier, C. J. et al. Mycobacteria manipulate macrophage recruitment through coordinated use of membrane lipids. Nature. 505, 218-222. (2014).

47 Gopinath, K., Moosa, A., Mizrahi, V. \& Warner, D. F. Vitamin B(12) metabolism in Mycobacterium tuberculosis. Future Microbiol 8, 1405-1418 (2013).

48 Young, D. B., Comas, I. \& de Carvalho, L. P. Phylogenetic analysis of vitamin B12related metabolism in Mycobacterium tuberculosis. Front Mol Biosci. 2, 6. (2015)

49 Danilchanka, O. et al. An outer membrane channel protein of Mycobacterium tuberculosis with exotoxin activity. Proc Natl Acad Sci U S A. 111, 6750-6755. (2014).

50 Delahay et al. The coiled-coil domain of EspA is essential for the assembly of the type III secretion translocon on the surface of enteropathogenic Escherichia coli. J Biol Chem. 274, 35969-35974.

\section{References Methods}

51 David, M., Dzamba, M., Lister, D., Ilie, L. \& Brudno, M. SHRiMP2: sensitive yet practical SHort Read Mapping. Bioinformatics. 27, 1011-1012. (2011).

52 Milne, I. et al. Using Tablet for visual exploration of second-generation sequencing data. Brief Bioinform. 14, 193-202. (2013).

53 Pouseele, H. \& Supply, P. Accurate whole-genome sequencing-based epidemiological surveillance of mycobacterium tuberculosis. Methods in Microbiology. doi:10.1016/bs.mim.2015.04.001 (2015).

54 Huson, D. H. \& Bryant, D. Application of phylogenetic networks in evolutionary studies. Mol Biol Evol 23, 254-267 (2006).

55 Brosch, R. et al. Comparative genomics uncovers large tandem chromosomal duplications in Mycobacterium bovis BCG Pasteur. Yeast 17, 111-123. (2000).

56 Brosch, R. et al. Use of a Mycobacterium tuberculosis H37Rv bacterial artificial chromosome library for genome mapping, sequencing, and comparative genomics. Infect Immun 66, 2221-2229 (1998).

57 Pham, T. T., Jacobs-Sera, D., Pedulla, M. L., Hendrix, R. W. \& Hatfull, G. F. Comparative genomic analysis of mycobacteriophage Tweety: evolutionary insights and construction of compatible site-specific integration vectors for mycobacteria. Microbiology. 153, 2711-2723 (2007). 
85558 Cascioferro, A. et al. Xer site-specific recombination, an efficient tool to introduce 856 unmarked deletions into mycobacteria. Appl Environ Microbiol 76, 5312-5316 (2010).

85759 Delogu, G. et al. Rv1818c-encoded PE_PGRS protein of Mycobacterium tuberculosis 858 is surface exposed and influences bacterial cell structure. Mol Microbiol 52, 725-733 859 (2004).

86060 Majlessi, L. et al. Influence of ESAT-6 Secretion System 1 (RD1) of Mycobacterium 861 862 tuberculosis on the Interaction between Mycobacteria and the Host Immune System. $J$ Immunol 174, 3570-3579 (2005).

863 


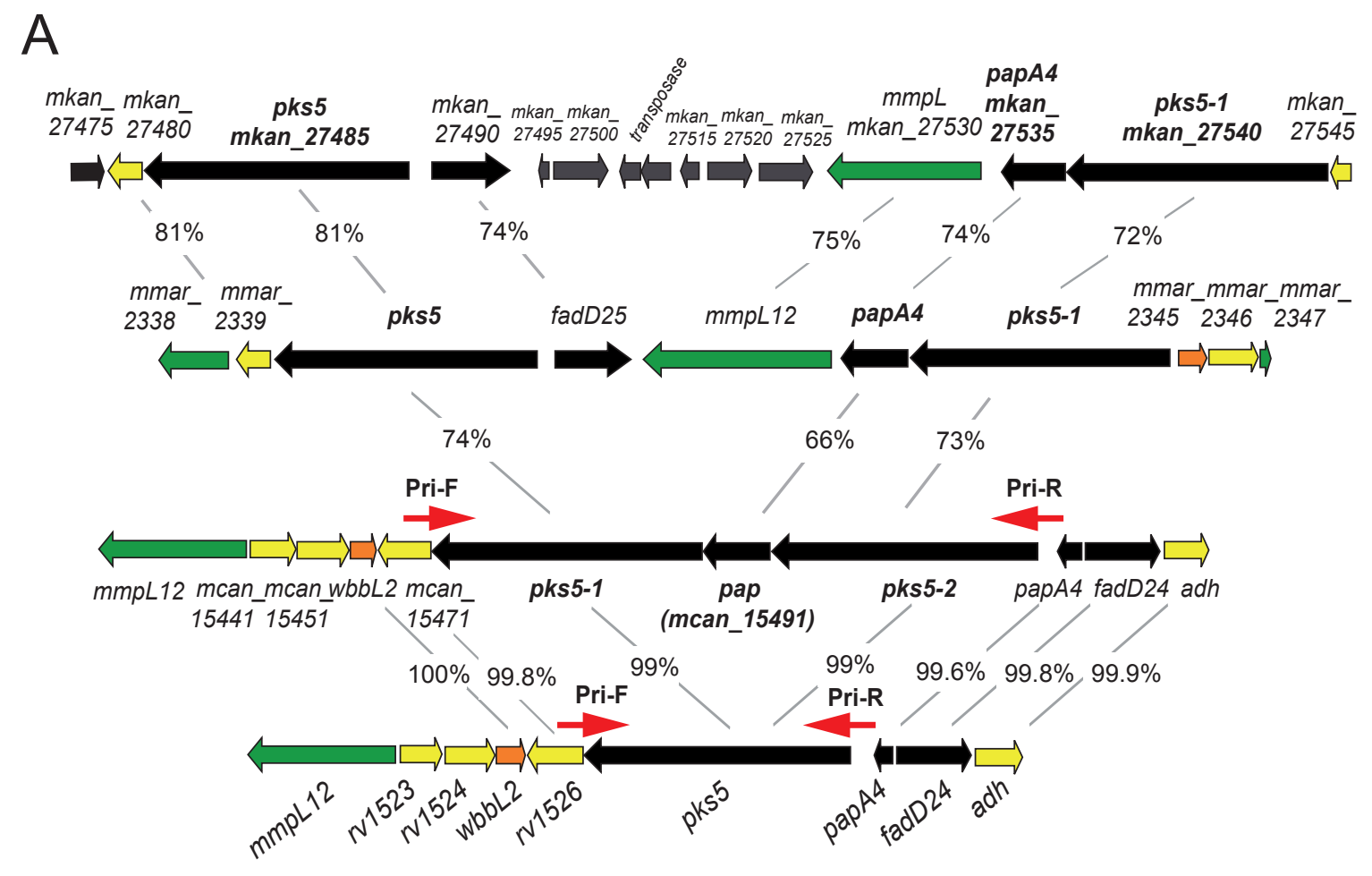

M. kansasii

ATCC 12478

M. marinum $\mathrm{M}$

M. canettii A

Mtb H37Rv

B

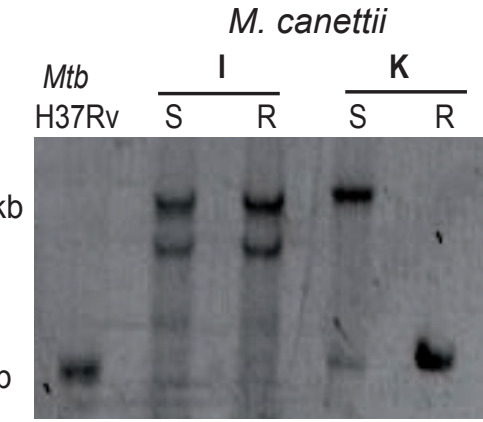

C

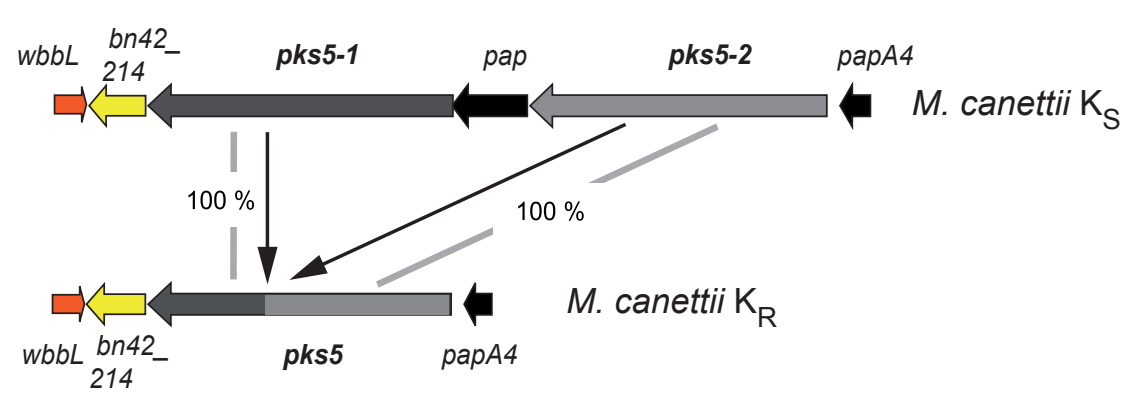

D M. canettii $\mathrm{K}_{\mathrm{S}}$

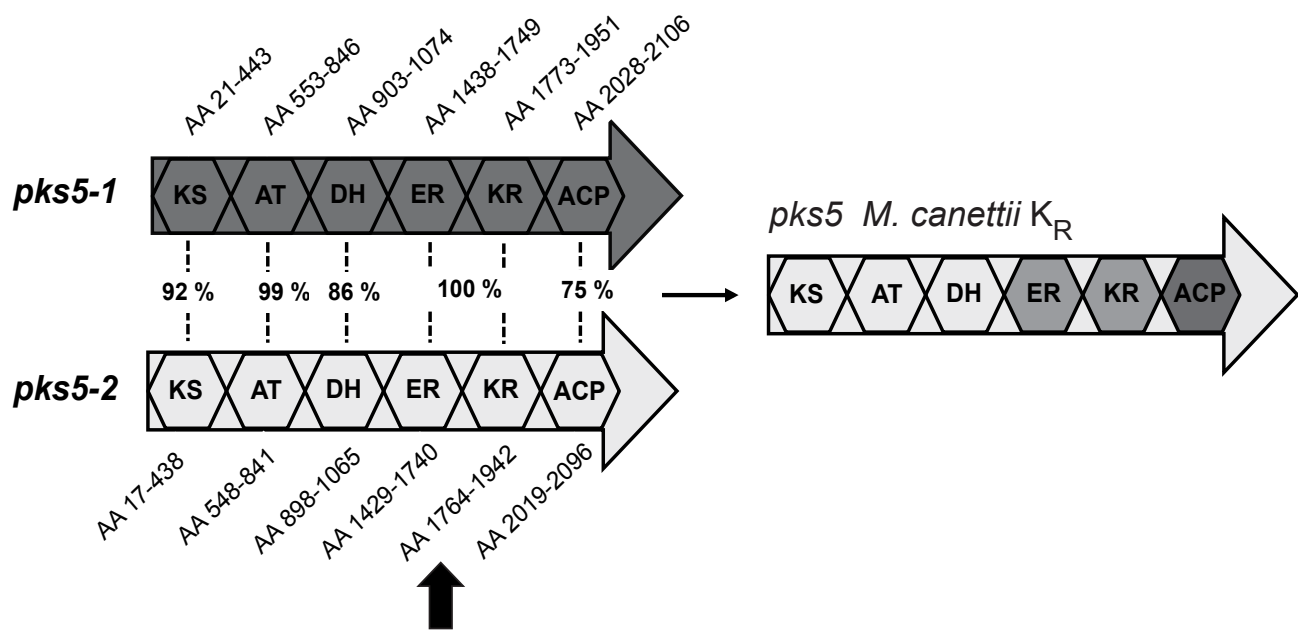

Region of recombination 
A
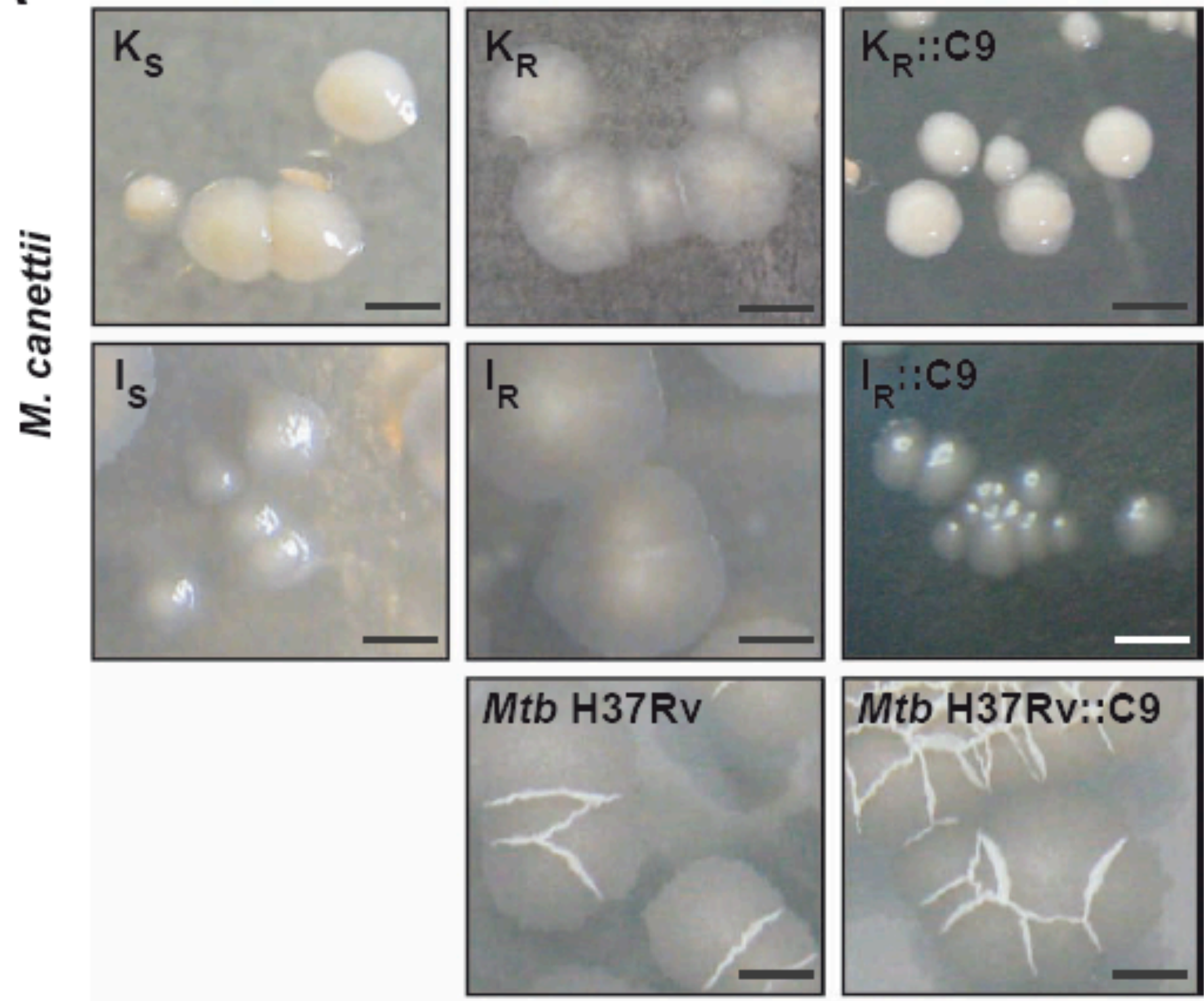

B
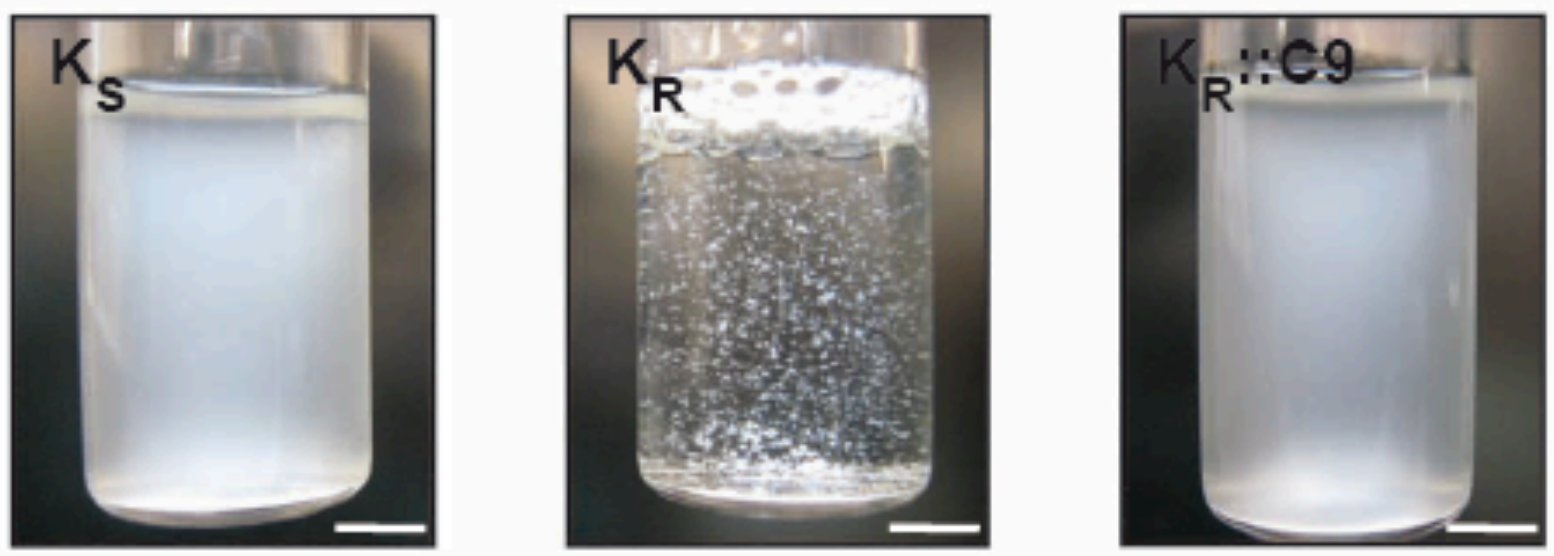

C
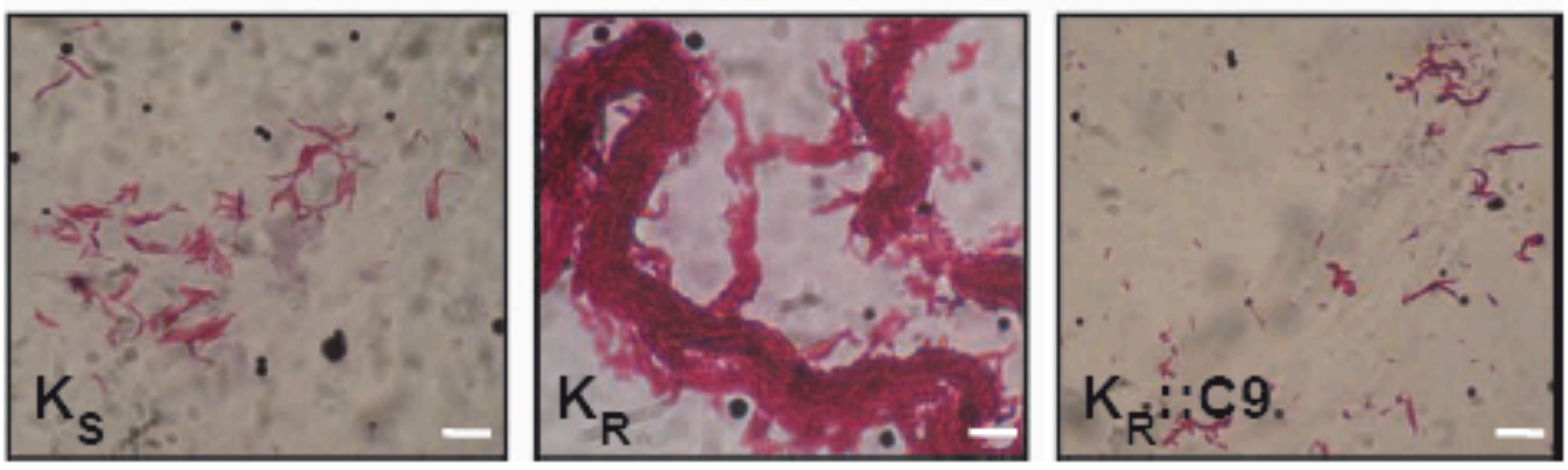

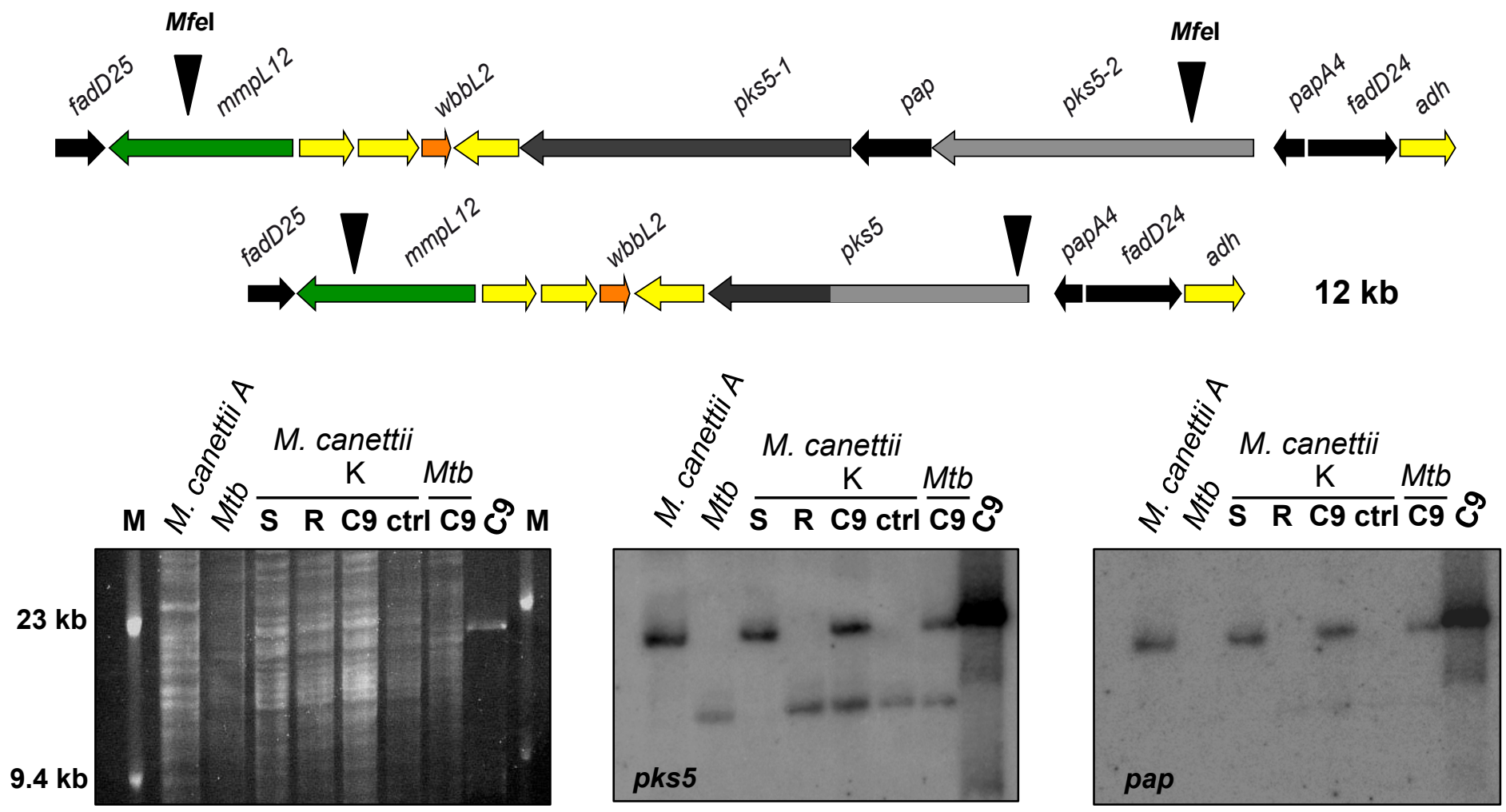
A

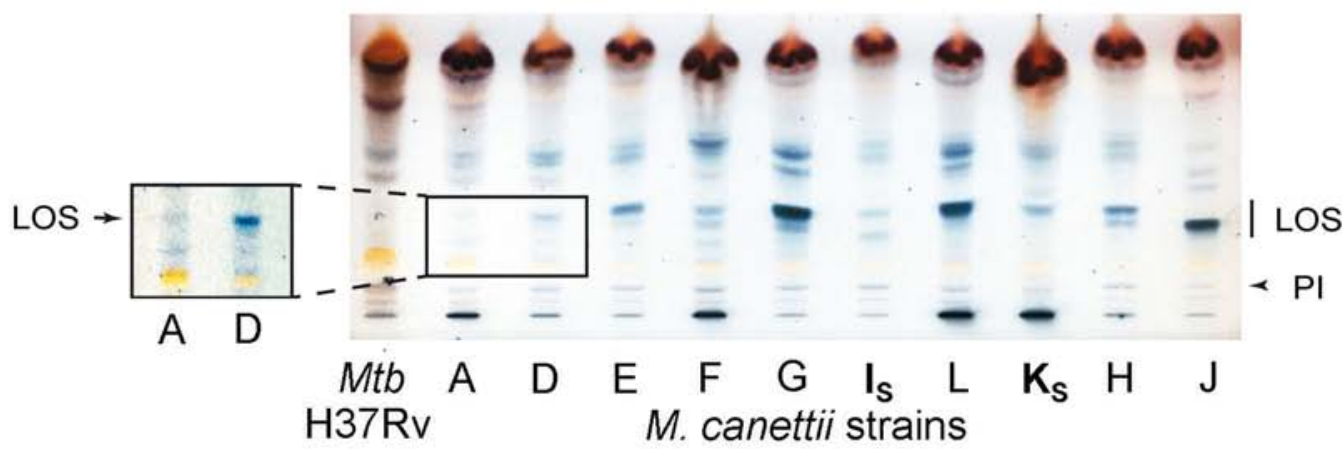

B

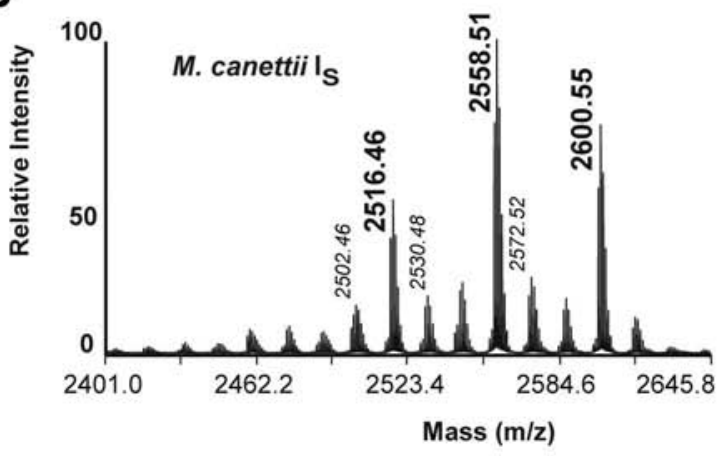

C
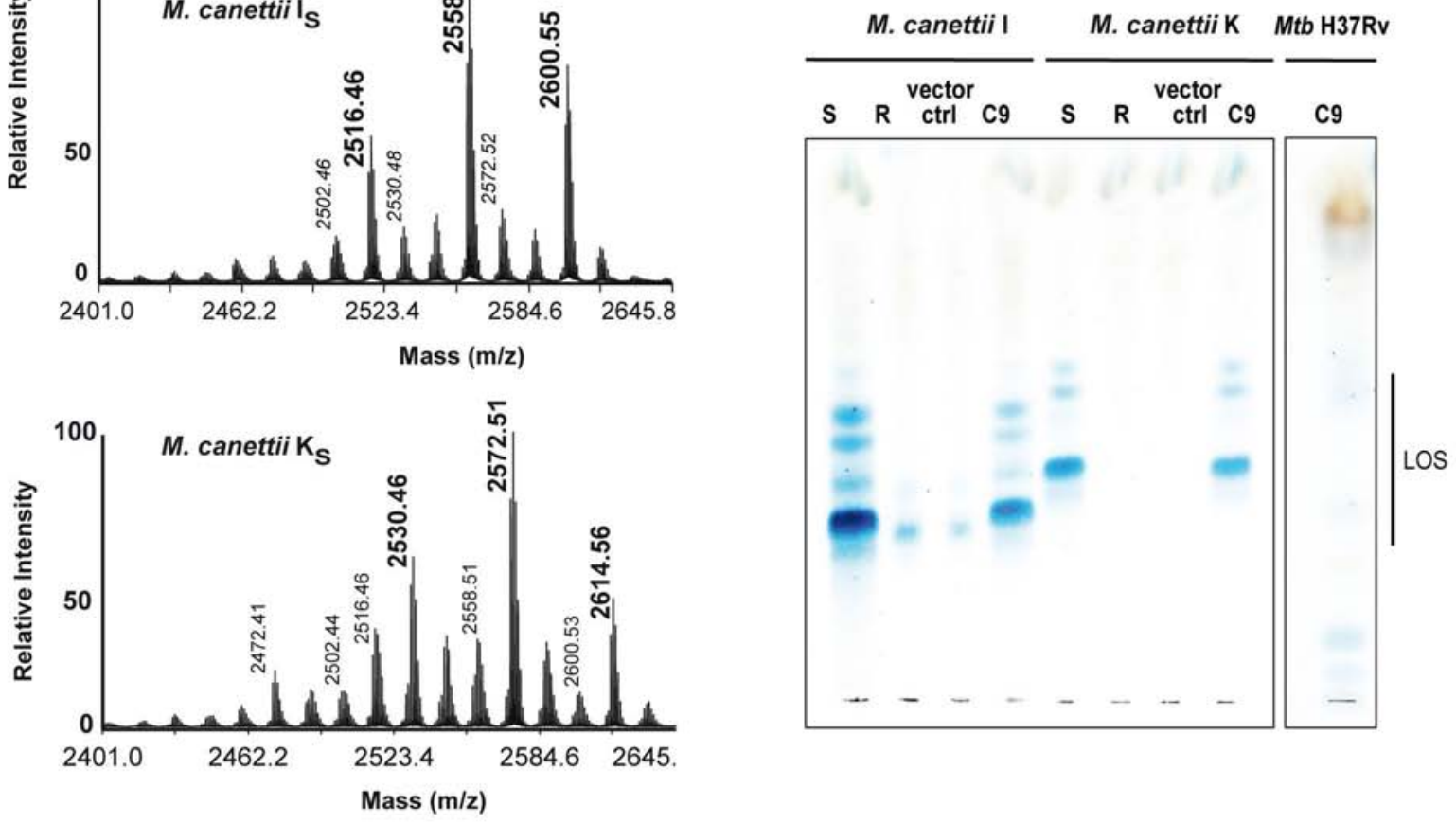

D

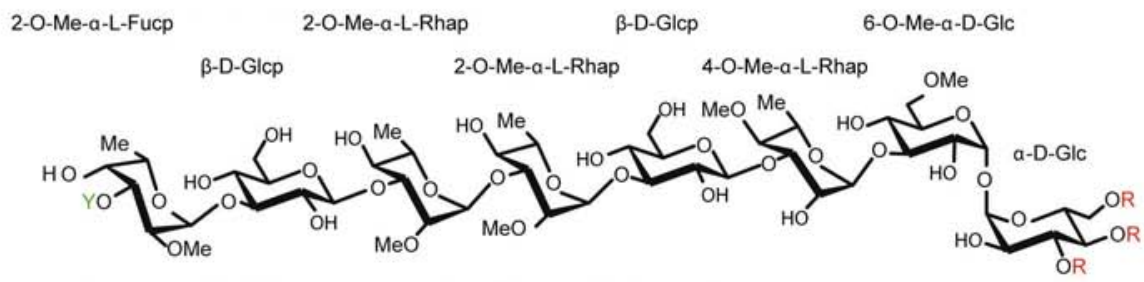

$\mathrm{Y}=$ incompletely defined $\mathrm{N}$-acyl derivatives of 4-amino-4,6-dideoxy-Galp<smiles>[R]=CCC(=O)C(C)C(O)C(C)(C)C</smiles>

2-methyl-3-hydroxyeicosanoate<smiles>CCC(=O)C(C)CC(C)C(C)C</smiles><smiles>CCC(=O)C(C)CC(C)CC(C)CC(C)C(C)(C)C</smiles>

2L-, 4L-dimethylhexadecanoate 
A

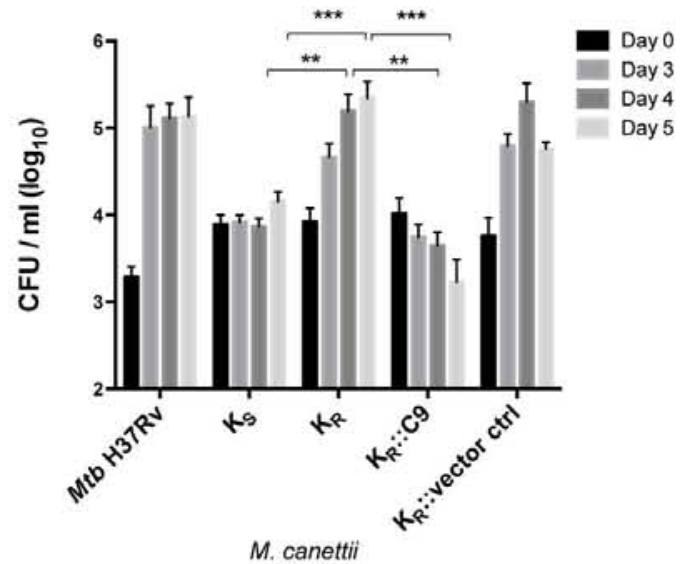

C

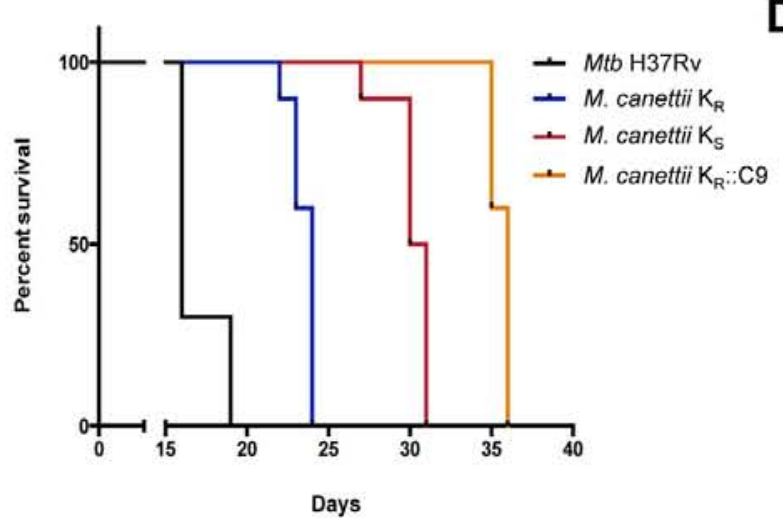

E

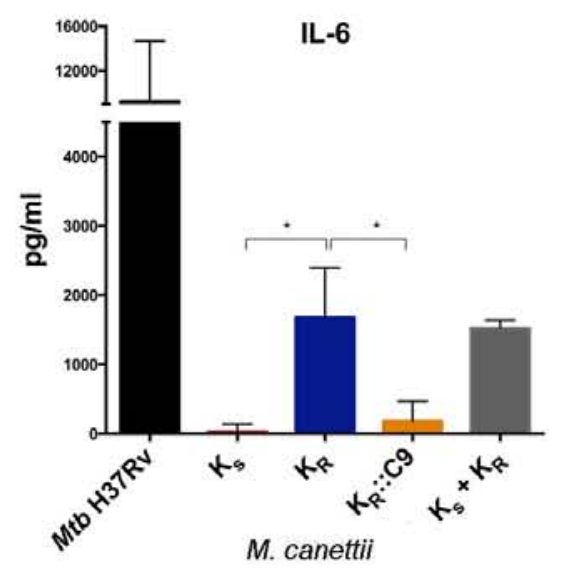

G

TLR2

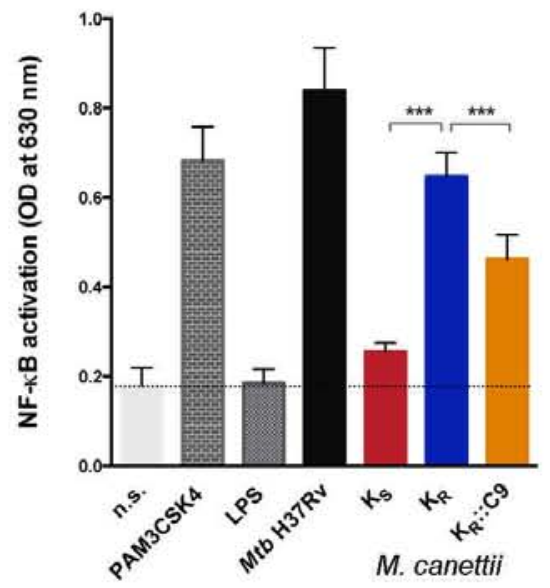

B

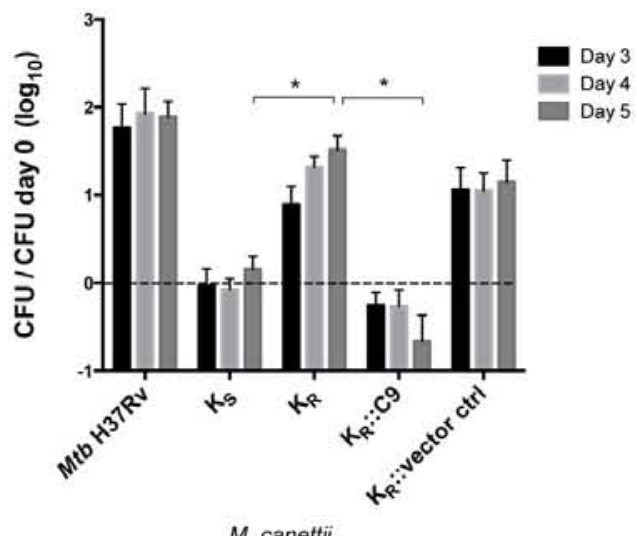

D

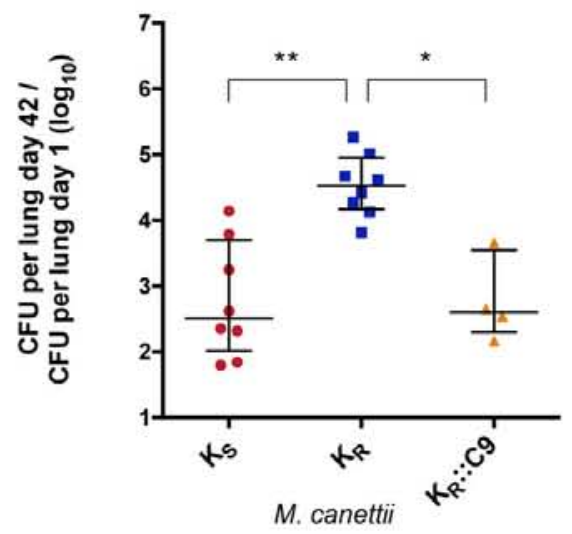

F

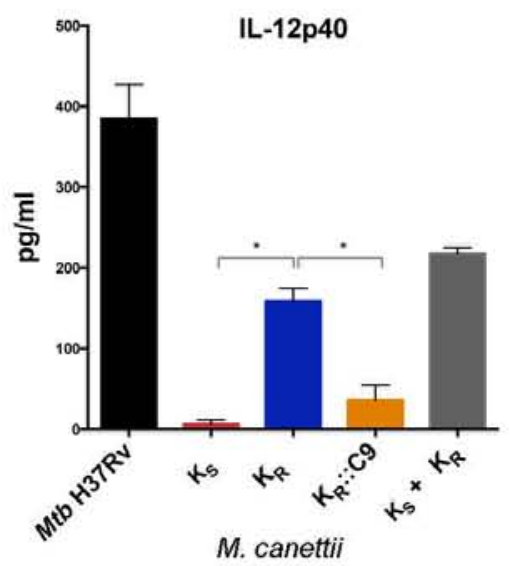

H TLR4

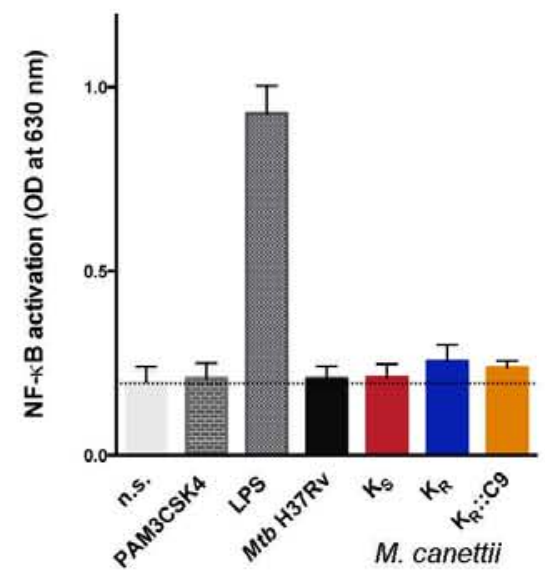


